\title{
Lifting Integrity Constraints in Binary Aggregation*
}

\author{
Umberto Grandi†and Ulle Endriss \\ Institute for Logic, Language and Computation \\ University of Amsterdam \\ Postbus 94242, 1090 GE Amsterdam \\ The Netherlands \\ umberto.uni@gmail.com, ulle.endriss@uva.nl
}

\begin{abstract}
We consider problems in which several individuals each need to make a yes/no choice regarding a number of issues and these choices then need to be aggregated into a collective choice. Depending on the application at hand, different combinations of yes/no may be considered rational. We describe rationality assumptions as integrity constraints using a simple propositional language and we explore the question of whether or not a given aggregation procedure will lift a given integrity constraint from the individual to the collective level, i.e., whether the collective choice will be rational whenever all individual choices are.

Keywords:collective decision making, computational social choice, multi-issue domains, combinatorial vote, judgment aggregation.
\end{abstract}

\section{Introduction}

Social Choice Theory (SCT) is the study of mathematical models for collective decision making. In recent times, this discipline has received increasing attention in Artificial Intelligence (AI), as testified by a large number of papers on social choice at the major AI conferences and by the creation of an entirely new research agenda under the name of Computational Social Choice (Chevaleyre et al., 2007). There are several good reasons for this trend. On the one hand, a number of methods developed in AI and, more generally, in Computer Science have turned out to be useful to deepen our understanding of social choice and, in some cases, can even suggest an entirely new perspective on classical problems. Examples include the complexity-theoretic analysis of optimisation problems arising in social choice (Faliszewski et al., 2009; Faliszewski and Procaccia, 2010) and the creation of new choice procedures inspired by classical techniques in knowledge representation (Lang et al., 2011). On the other hand, methods from SCT have natural important applications in AI. They can, e.g., be employed to achieve consensus amongst the autonomous software agents in a multiagent system (Shoham and Leyton-Brown, 2009), to aggregate the output of several search engines (Altman and Tennenholtz, 2008), or to inform the design of online recommender systems (Pennock et al., 2000). One particular problem of interest for AI is the case of social choice in combinatorial domains, in which the space of alternatives from which the individuals have to choose has a multi-attribute structure (Lang, 2004; Chevaleyre et al., 2008). Classical examples include voting in multiple referenda, where we have to decide which of a set of propositions to accept, or electing a committee, where we have to decide how to fill each seat. There have been several attempts to tackle the high complexity that arises in this context by using tools from AI, such as methods for

\footnotetext{
*To appear on Artificial Intelligence. Accepted in May 2013.

${ }^{\dagger}$ Corresponding author. Current affiliation: Department of Mathematics, University of Padova, Via Trieste 63, 35121 Padova, Italy. Tel: +39 (0) 498271357.
} 
modelling preferences inspired by knowledge representation (Lang and Xia, 2009; Rossi et al., 2004). Finally, SCT provides tools for the analysis of collective choices of groups of agents, and as such is of immediate relevance to the study of multiagent systems.

A central problem in SCT, and, in view of our previous discussion, in all its applications to AI, is the problem of aggregation: Suppose a group of agents each supply a particular piece of information regarding a common problem and we want to aggregate this information into a collective view to obtain a summary of the individual views provided. A classical example is that of preferences (Arrow, 1963): each agent declares their individual preferences over a set of alternatives by providing an ordering over this set, and we are asked to amalgamate this information into a collective ranking that represents the individual preferences provided. The same methodology has also been applied more recently to a number of other types of information, such as beliefs (Konieczny and Pino Pérez, 2002, 2011) and judgments (List and Puppe, 2009).

One of the main features of the study of aggregation is the problem of collective rationality: given a rationality assumption that bounds the choices of the individuals, we ask whether the output of an aggregator still satisfies the same rationality assumption. To understand this problem better, consider the following example: three autonomous agents need to decide on whether to perform a collective action. This action is performed if two parameters are estimated to exceed a certain threshold. We can model the choice situation with a multi-attribute domain in which there are three issues at stake: "the first parameter is above the threshold" $\left(T_{1}\right)$, "the second parameter is above the threshold" $\left(T_{2}\right)$, and "the action should be performed" $(A)$. The rationality assumption that links the three issues together can be modelled using a simple propositional formula, namely $T_{1} \wedge T_{2} \rightarrow A$. The individual views on the three issues are then aggregated using the majority rule, which accepts an issue if a majority of the individual agents do. Consider now the following situation:

\begin{tabular}{lccc} 
& $T_{1}$ & $T_{2}$ & $A$ \\
\hline Agent 1: & Yes & Yes & Yes \\
Agent 2: & No & Yes & No \\
Agent 3: & Yes & No & No \\
\hline Majority: & Yes & Yes & No
\end{tabular}

In the situation described above the collective action $A$ is not performed, even though a majority of the individuals think that the first parameter exceeds the threshold and a (different) majority agree that also the second parameter exceeds the threshold. Situations like the one above are considered paradoxical: even if each individual agent is rational (i.e., each of them satisfies the rationality assumption), the collective view derived using the majority rule is not. That is, the majority rule fails to lift the integrity constraint $T_{1} \wedge T_{2} \rightarrow A$ from the individual to the collective level. This example shows that the majority rule violates collective rationality in certain specific cases. Similar examples can be devised for a number of different situations ranging from voting to rank aggregation, to the development of a collective judgment in court cases.

Classical work in SCT was restricted to particular studies of collective rationality in a given aggregation situation and for a given class of aggregation procedures. Dokow and Holzman (2010a), for instance, characterise binary domains of aggregation over which every procedure that satisfies certain desirable axiomatic properties, namely, independence and unanimity, is dictatorial (see Section 8). This is a good example for the use of the axiomatic method in economic theory: the aim is to identify the appropriate set of axiomatic properties (e.g., to model real-world economies, specific moral ideals, etc.) and then to prove a characterisation (or impossibility) result for those axioms. Given the wide variety of potential applications in AI, on the other hand, in this context we require instead a systematic study that, depending on the situation at hand, can give answers to the problem of collective rationality. With every new application the principles underlying a system may change; so we may be more interested in devising languages for expressing a range of different axiomatic properties rather than identifying the "right" set of axioms; and we may be more interested in developing methods that will help us to understand the dynamics of a range of different social choice scenarios rather than in technical results for a specific such scenario. 
In this paper we put forward a general framework that encompasses most of the classical studies of collective rationality in SCT, and that can prove useful to diverse research areas in AI. We base our framework on binary aggregation, in which individuals are required to choose from a multi-issue domain where issues represent different binary choices. Classical frameworks for the study of aggregation, such as preference and judgment aggregation, can be embedded in this framework. We model rationality assumptions using a simple propositional language, and we give a precise definition of collective rationality with respect to a given rationality assumption. We classify rationality assumptions with respect to their syntactic properties, and we give a systematic treatment of the question of how to relate collective rationality with respect to a syntactically defined sublanguage to classical axiomatic properties from $\mathrm{SCT}$. For instance, we have already seen that the majority rule is not collectively rational with respect to the integrity constraint $T_{1} \wedge T_{2} \rightarrow A$. It is also not collectively rational with respect to the 3 -clause $T_{1} \vee T_{2} \vee A$ : to see this, consider a scenario with three agents, where each agent accepts exactly one issue, and no two agents accept the same issue. On the other hand, as we shall see, any 2-clause will always be lifted, i.e., the majority rule is collectively rational with respect to the language of 2-clauses. We will then be able to describe the majority rule in terms of classical axioms (see Proposition 2) or in terms of the subset of integrity constraints it lifts (see Theorem 28). It is results of this kind that we shall explore in depth in this paper, establishing a link between standard axiomatic requirements from $\mathrm{SCT}$ and collective rationality with respect to fragments of the propositional language.

This paper expands our initial work on this topic (Grandi and Endriss, 2010), complementing it with further results from previous work (Grandi and Endriss, 2011; Grandi, 2012b,a).

The paper is organised as follows. We begin by defining the basic notions that constitute the framework of binary aggregation with integrity constraints in Section 2. In this section, we give the crucial definition of collective rationality with respect to an integrity constraint expressed in a suitable propositional language, and we list several axiomatic properties adapted from the literature on SCT. We also provide several examples of aggregation problems that can be modelled in binary aggregation with integrity constraints, such as multi-issue elections, preference aggregation, judgment aggregation, and the problem of choosing from a set of candidates. In Section 3 we define the notion of language for integrity constraints, and we prove this to be the correct definition for the study of collective rationality (see Lemma 6). We then define classes of aggregation procedures both in terms of collective rationality and by using classical axiomatic requirements. We study the properties of these two means of defining classes of procedures, developing a theoretical machinery that enables us to provide a systematic treatment of the relation between propositional languages for integrity constraints and axiomatic properties. This is done in the two following sections. In Section 4 we characterise in axiomatic terms, for several fragments of the propositional language, the class of collectively rational procedures with respect to formulas in this fragment. In Section 5 we start from classical axiomatic properties, and we explore to what extent classes of aggregation procedures defined in these terms can be characterised as classes of collectively rational procedures for a given language. We provide negative results for three important axioms taken from the literature on SCT. These three axioms can be combined to obtain an interesting class of procedures known as quota rules, defined by the choice of an acceptance quota for every issue. In Section 6 we concentrate on this particular class. We give precise bounds on the acceptance quotas to guarantee collective rationality with respect to integrity constraints that belong to given languages of clauses, i.e., disjunctions of literals. This includes a characterisation of the set of integrity constraints that are lifted by the majority rule (see Theorem 28). We discuss the significance of our results and their relation to classical frameworks of aggregation in Section 7. Related work is discussed in Section 8 and Section 9 concludes.

\section{Basic Definitions}

In this section we give the basic definitions that constitute the framework of binary aggregation with integrity constraints. First, we review the classical setting of binary aggregation, based on work by Wilson (1975) and Dokow and Holzman (2010a), to which we add the notion of integrity constraint as 
a mean of specifying the set of individual ballots to be considered rational. We then present a list of examples from both practical applications and classical frameworks of aggregation that can be modelled in our framework. Finally, we state the crucial definition of collective rationality and we review the classical axiomatic method for binary aggregation procedures. We conclude by axiomatising the class of quota rules and the majority rule over binary combinatorial domains.

\subsection{Binary Aggregation with Integrity Constraints}

Let $\mathcal{I}=\{1, \ldots, m\}$ be a finite set of issues, and let $\mathcal{D}=D_{1} \times \cdots \times D_{m}$ be a boolean combinatorial domain, i.e., $\left|D_{i}\right|=2$ for all $i \in \mathcal{I}$ (we assume $D_{i}=\{0,1\}$ ). Let $\mathcal{N}=\{1, \ldots, n\}$ be a finite set of individuals and we assume $|\mathcal{N}|>1$. A ballot $B$ is an element of $\mathcal{D}$. A profile $\boldsymbol{B}=\left(B_{1}, \ldots, B_{n}\right)$ is a vector of ballots, one for each individual in $\mathcal{N}$. We write $b_{j}$ for the $j$ th element of a ballot $B$, and $b_{i, j}$ for the $j$ th element of ballot $B_{i}$ within a profile $\boldsymbol{B}=\left(B_{1}, \ldots, B_{n}\right)$.

Definition 1. Given a finite set of issues $\mathcal{I}$ and a finite set of individuals $\mathcal{N}$, an aggregation procedure is a function $F: \mathcal{D}^{\mathcal{N}} \rightarrow \mathcal{D}$, mapping each profile of binary ballots to an element of $\mathcal{D} .^{1}$

Let $F(\boldsymbol{B})_{j}$ denote the result of the aggregation on issue $j$. Note that we do not put any restriction on the domain on which aggregation procedures are defined, i.e., Definition 1 includes a requirement known as universal domain.

If $\mathcal{I}$ is a set of $m$ issues, let $P S=\left\{p_{1}, \ldots, p_{m}\right\}$ be a set of propositional symbols, one for each issue, and let $\mathcal{L}_{P S}$ be the propositional language constructed by closing $P S$ under propositional connectives. For any formula $\varphi \in \mathcal{L}_{P S}$, let $\operatorname{Mod}(\varphi)$ be the set of assignments that satisfy $\varphi$. For example, $\operatorname{Mod}\left(p_{1} \wedge \neg p_{2}\right)=$ $\{(1,0,0),(1,0,1)\}$ if $P S=\left\{p_{1}, p_{2}, p_{3}\right\}$.

Definition 2. An integrity constraint is any formula $\mathrm{IC} \in \mathcal{L}_{P S}$.

Integrity constraints can be used to define what tuples in $\mathcal{D}$ we consider rational choices. Any ballot $B \in \mathcal{D}$ is an assignment to the variables $p_{1}, \ldots, p_{m}$, and we call $B$ a rational ballot if it satisfies the integrity constraint IC, i.e., if $B$ is an element of $\operatorname{Mod}(\mathrm{IC})$. A rational profile will be therefore an element of $\operatorname{Mod}(\mathrm{IC})^{\mathcal{N}}$. In the sequel we shall use the terms "integrity constraints" and "rationality assumptions" interchangeably.

\section{$2.2 \quad$ Examples}

In line with the example presented in the introduction, let us consider several other aggregation problems that can be modelled in binary aggregation by devising a suitable integrity constraint:

Example 1. (Multi-issue elections under constraints) A committee $\mathcal{N}$ has to decide on each of the three following issues: $(U)$ financing a new university building, $(S)$ financing a sports centre, $(C)$ increasing catering facilities. As an approval of both a new university building and a sports centre would bring an unsustainable demand on current catering facilities, it is considered irrational to approve both of the first two issues and to reject the third one. We can model this situation with a set of three issues $\mathcal{I}=\{U, S, C\}$. The integrity constraint representing this rationality assumption is the following formula: $p_{U} \wedge p_{S} \rightarrow p_{C}$. To see an example of a rational profile, consider the situation described in Table 1 for the case of a committee with three members. All individuals are rational, the only irrational ballot being $B=(1,1,0)$.

\footnotetext{
${ }^{1}$ In this definition we represent profiles as functions from the set of individuals $\mathcal{N}$ to the domain $\mathcal{D}$, indicating the set of all profiles with $\mathcal{D}^{\mathcal{N}}$. Given that in this paper the set of individuals is finite, profiles can also be represented as tuples of binary ballots. However, we decided to use the functional notation to facilitate an eventual generalisation of the framework to allow for infinite sets of individuals.
} 


\begin{tabular}{|c|c|c|c|c|}
\hline \multirow{4}{*}{$B$} & & $U$ & $S$ & $C$ \\
\hline & $i_{1}$ & 0 & 1 & 0 \\
\hline & $i_{2}$ & 1 & 0 & \\
\hline & $i_{3}$ & 1 & 1 & 1 \\
\hline
\end{tabular}

Table 1: A rational profile for $p_{U} \wedge p_{S} \rightarrow p_{C}$.

Example 2. (Preference aggregation) A set $\mathcal{N}$ of individuals has to agree on a ranking of three alternatives $a, b$ and $c$. Each individual submits its own ranking of the alternatives from the most preferred to the least preferred, e.g., $b>a>c$. We can model this situation using a binary issue for every pair of alternatives: issue $a b$ stands for "alternative $a$ is preferred to alternative $b "$ ". The set of issues is therefore $\mathcal{I}=\{a a, b b, c c, a b, b a, b c, c b, a c, c a\}$. However, not every binary evaluation over this set of issues corresponds to a ranking. An integrity constraint needs to be devised to encode the correct properties of the binary relation: transitivity, completeness and irreflexivity. This can be done by considering the following set of integrity constraints $\mathrm{IC}_{<}$:

Irreflexivity: $\neg p_{x x}$ for all $x \in\{a, b, c\}$

Completeness: $p_{x y} \vee p_{y x}$ for all $x, y \in\{a, b, c\}$ distinct

Transitivity: $p_{x y} \wedge p_{y z} \rightarrow p_{x z}$ for $x, y, z \in\{a, b, c\}$ pairwise distinct

These formulas can be easily modified to account for different representations of preferences that are closer to applications in AI, e.g., partially ordered preferences (Pini et al., 2009).

Example 3. (Judgment aggregation) A court composed of three judges has to decide on the liability of a defendant under the charge of breach of contract. According to the law, the individual is liable if there was a valid contract and her behaviour was such as to be considered a breach of the contract. ${ }^{2}$ The court takes three majority decisions on the following statements: there was a valid contract $(\alpha)$, the individual broke the contract $(\beta)$, the defendant is liable $(\alpha \wedge \beta)$. We can model this situation using a set of six issues $\mathcal{I}=\{\alpha, \neg \alpha, \beta, \neg \beta, \alpha \wedge$ $\beta, \neg(\alpha \wedge \beta)\}$ to model the decision of a judge on the three issues at stake, and create a set of integrity constraints that reflect the consistency of a possible verdict by explicitly ruling out every inconsistent set that can be created using issues in $\mathcal{I}$ :

Inconsistent sets of size 2: $\neg\left(p_{x} \wedge p_{\neg x}\right)$ for all $x \in\{\alpha, \beta, \alpha \wedge \beta\}$,

$$
\neg\left(p_{\alpha \wedge \beta} \wedge p_{\neg \alpha}\right) \text { and } \neg\left(p_{\alpha \wedge \beta} \wedge p_{\neg \beta}\right)
$$

Inconsistent set of size 3: $\quad \neg\left(p_{\neg(\alpha \wedge \beta)} \wedge p_{\alpha} \wedge p_{\beta}\right)$

Situations like the one described in this example are the subject of a wide literature in SCT under the name of judgment aggregation (List and Puppe, 2009). We refer to our previous work (Grandi and Endriss, 2011; Grandi, 2012a) for more details about the correspondence between judgment aggregation and our framework of binary aggregation with integrity constraints.

Example 4. (Choosing from a set of candidates) A winning candidate has to be chosen from a set $C=\{1, \ldots, m\}$ by an electorate $\mathcal{N}$. Let the set of issues be $\mathcal{I}=C$. If we are using an aggregation procedure like approval voting (Brams and Fishburn, 2007), in which individuals are submitting a set of candidates they approve of, then we can model the situation without any integrity constraint, since every binary ballot over $\mathcal{I}$ corresponds to a set of candidates. If instead we want to consider more restrictive ballots, like in the case of the plurality rule in which each individual submits only its favourite candidate, then we need to devise an integrity

\footnotetext{
${ }^{2}$ This example is due to Kornhauser and Sager (1986).
} 
constraint that forces each individual to approve a single candidate in the list. This can be done by taking the disjunction of all possible ballots:

$$
\left(p_{1} \vee \neg p_{2} \wedge \cdots \wedge \neg p_{m}\right) \vee\left(\neg p_{1} \wedge p_{2} \wedge \cdots \wedge \neg p_{m}\right) \vee \cdots \vee\left(\neg p_{1} \wedge \cdots \wedge \neg p_{m-1} \wedge p_{m}\right)
$$

The voting rule known as $k$-approval voting, in which individuals submit a set of $k$ approved candidates, can be modelled in a similar fashion.

\subsection{Collective Rationality}

Consider the situation introduced in Example 1: There are three issues at stake, and the integrity constraint is represented by the formula IC $=p_{U} \wedge p_{S} \rightarrow p_{C}$. Suppose there are three individuals, choosing ballots $(0,1,0),(1,0,0)$ and $(1,1,1)$, as in Table 1 . Their choices are rational (they all satisfy IC). Assume now we accept an issue $j$ if a majority of individuals do, employing what we will call the majority rule. We would then obtain as an outcome of this profile the ballot $(1,1,0)$, which fails to be rational. This kind of observation, like the one presented in the introduction, is often referred to as a paradox.

In the literature on SCT, situations like the one above are ruled out by requiring aggregation procedures to satisfy a property called collective rationality, which forces the output of an aggregation procedure to be of the same form of the input, i.e., a rational ballot. In preference aggregation, for instance, the output of an aggregation procedure is required to be a linear (or weak) order over a set of alternatives (Arrow, 1963). In judgment aggregation the output is required to be a consistent judgment over a set of propositional formulas (List and Puppe, 2009). Here we give a general definition of collective rationality depending on the integrity constraint at hand:

Definition 3. Given an integrity constraint IC $\in \mathcal{L}_{P S}$, an aggregation procedure $F: \mathcal{D}^{\mathcal{N}} \rightarrow \mathcal{D}$ is called collectively rational $(C R)$ with respect to $\mathrm{IC}$, if for all rational profiles $\boldsymbol{B} \in \operatorname{Mod}(\mathrm{IC})^{\mathcal{N}}$ we have that $F(\boldsymbol{B}) \in \operatorname{Mod}(\mathrm{IC})$.

Thus, $F$ is CR if it lifts the rationality assumptions given by IC from the individual to the collective level. An aggregation procedure that is $\mathrm{CR}$ with respect to IC cannot generate a paradox with IC as integrity constraint.

Inspired by Definition 3, we can give a general definition of paradoxical behaviour of an aggregation procedure in terms of the violation of certain rationality assumptions:

Definition 4. A paradox is a triple $(F, \boldsymbol{B}, \mathrm{IC})$, where $F: \mathcal{D}^{\mathcal{N}} \rightarrow \mathcal{D}$ is an aggregation procedure, $\boldsymbol{B}$ is a profile in $\mathcal{D}^{\mathcal{N}}$, IC is an integrity constraint in $\mathcal{L}_{P S}$, and $B_{i} \in \operatorname{Mod}(\mathrm{IC})$ for all $i \in \mathcal{N}$ but $F(\boldsymbol{B}) \notin \operatorname{Mod}(\mathrm{IC})$.

This notion of paradox encompasses many of the classical paradoxes of aggregation studied in SCT. A shown by the examples presented in the previous section, preference and judgment aggregation, as well as other frameworks for collective decision making, can be modelled in binary aggregation by devising suitable integrity constraints. In previous work (Grandi, 2012b) we bring this correspondence one step further by showing how paradoxical situations traditionally studied in SCT, such as the Condorcet paradox in preference aggregation (Arrow, 1963) and the discursive dilemma in judgment aggregation (List and Puppe, 2009), can be viewed as particular instances of our Definition 4.

\subsection{The Axiomatic Method}

Aggregation procedures are traditionally studied using the axiomatic method. Axioms are used to express desirable properties of a procedure, depending on the application at hand. In this section, we adapt the most important axioms familiar from standard SCT, and more specifically from judgment aggregation (List and Puppe, 2009) and binary aggregation (Dokow and Holzman, 2010a), to our setting. Let $\mathcal{X} \subseteq \mathcal{D}^{\mathcal{N}}$ be a subset of the set of all profiles. We start with four common axioms: 
Unanimity (U): For any profile $\boldsymbol{B} \in \mathcal{X}$ and any $x \in\{0,1\}$, if $b_{i, j}=x$ for all $i \in \mathcal{N}$, then $F(\boldsymbol{B})_{j}=x$.

Issue-Neutrality $\left(\mathrm{N}^{\mathcal{I}}\right)$ : For any two issues $j, j^{\prime} \in \mathcal{I}$ and any profile $\boldsymbol{B} \in \mathcal{X}$, if for all $i \in \mathcal{N}$ we have that $b_{i, j}=b_{i, j^{\prime}}$, then $F(\boldsymbol{B})_{j}=F(\boldsymbol{B})_{j^{\prime}}$.

Independence (I): For any issue $j \in \mathcal{I}$ and any two profiles $\boldsymbol{B}, \boldsymbol{B}^{\prime} \in \mathcal{X}$, if $b_{i, j}=b_{i, j}^{\prime}$ for all $i \in \mathcal{N}$, then $F(\boldsymbol{B})_{j}=F\left(\boldsymbol{B}^{\prime}\right)_{j}$.

Anonymity (A): For any profile $\boldsymbol{B} \in \mathcal{X}$ and any permutation $\sigma: \mathcal{N} \rightarrow \mathcal{N}$, we have that $F\left(B_{1}, \ldots, B_{n}\right)=$ $F\left(B_{\sigma(1)}, \ldots, B_{\sigma(n)}\right)$.

Unanimity postulates that, if all individuals agree on issue $j$, then the aggregation procedure should implement that choice for $j$. Anonymity requires the procedure to be symmetric with respect to individuals. Issue-neutrality (a variant of the standard axiom of neutrality introduced in the literature on judgment aggregation) asks that the procedure be symmetric with respect to issues. Finally, independence requires the outcome of aggregation on a certain issue $j$ to depend only on the individual choices regarding that issue.

It is important to remark that all axioms are domain-dependent, as testified by the domain restriction given by $\mathcal{X}$. For instance, many aggregation procedures, such as the majority rule, are independent over the full set of profiles $\mathcal{X}=\mathcal{D}^{\mathcal{N}}$, while others, such as the one presented in the next example, are not. With two issues, let IC $=\left(p_{2} \rightarrow p_{1}\right)$ and let $F$ be equal to the majority rule on the first issue, and accept the second issue only if the first one was accepted and the second one has the support of a majority of the individuals. This procedure is not independent on the full domain, but it is easy to see that it satisfies independence when restricted to $\mathcal{X}=\operatorname{Mod}(\mathrm{IC})^{\mathcal{N}}$. As in the previous example, in the following sections we will specify restrictions on the domain of aggregation by means of propositional formulas.

As a generalisation of the axiom of neutrality introduced by May (1952), we introduce the following axiom:

Domain-Neutrality $\left(\mathrm{N}^{\mathcal{D}}\right)$ : For any two issues $j, j^{\prime} \in \mathcal{I}$ and any profile $\boldsymbol{B} \in \mathcal{X}$, if $b_{i, j}=1-b_{i, j^{\prime}}$ for all $i \in \mathcal{N}$, then $F(\boldsymbol{B})_{j}=1-F(\boldsymbol{B})_{j^{\prime}}$.

The two notions of neutrality are uncorrelated but dual: issue-neutrality requires the outcome on two issues to be the same if all individuals agree on these issues; domain-neutrality requires it to be reversed if all the individuals make opposite choices on the two issues.

We now introduce two axioms of monotonicity. The first, which we call independence-monotonicity, is often called positive responsiveness and is formulated as an (inter-profile) axiom for independent aggregation procedures. The second version of monotonicity is designed for neutral procedures, and it was introduced in our previous work (Endriss et al., 2010):

I-Monotonicity $\left(\mathrm{M}^{\mathrm{I}}\right)$ : For any $j \in \mathcal{I}$ and any two profiles $\boldsymbol{B}$ and $\boldsymbol{B}^{\prime}$ in $\mathcal{X}$, if $b_{i, j}=1$ entails $b_{i, j}^{\prime}=1$ for all $i \in \mathcal{N}$, and for some $s \in \mathcal{N}$ we have that $b_{s, j}=0$ and $b_{s, j}^{\prime}=1$, then $F(\boldsymbol{B})_{j}=1$ entails $F\left(\boldsymbol{B}^{\prime}\right)_{j}=1$.

N-Monotonicity $\left(\mathrm{M}^{\mathrm{N}}\right)$ : For any $j, j^{\prime} \in \mathcal{I}$ and profile $\boldsymbol{B} \in \mathcal{X}$, if for all $i \in \mathcal{N}$ we have that $b_{i, j}=1$ entails $b_{i, j^{\prime}}=1$ and for some $s \in \mathcal{N}$ we have that $b_{s, j}=0$ and $b_{s, j^{\prime}}=1$, then $F(\boldsymbol{B})_{j}=1$ entails $F(\boldsymbol{B})_{j^{\prime}}=1$.

That is, $\mathrm{M}^{\mathrm{I}}$ expresses that if an issue $j$ is collectively accepted and receives additional support (from an individual $i$ ), then it should continue to be collectively accepted. Axiom $\mathrm{M}^{\mathrm{N}}$ says that if issue $j$ is collectively accepted and issue $j^{\prime}$ is accepted by a strict superset of the individuals accepting $j$, then $j^{\prime}$ should also be collectively accepted.

The last property for aggregation procedures that we are going to introduce is traditionally considered a negative one. We choose not to state it as an axiom, but rather as a property defining a class of functions: An aggregation procedure is called a dictatorship if it copies the ballot of the same individual in every 
profile. This notion is in clear contrast with the axiom of anonymity previously introduced. In Definition 9 we will generalise this notion by defining the class of generalised dictatorships as those procedures that copy the ballot of a possibly different individual in every profile.

\subsection{Quota Rules}

An aggregation procedure $F$ for $n$ individuals is a quota rule if for every issue $j$ there exists a quota $0 \leqslant q_{j} \leqslant n+1$ such that, if we denote by $N_{j}^{B}=\left\{i \mid \boldsymbol{B}_{i, j}=1\right\}$, then $F(\boldsymbol{B})_{j}=1$ if and only if $\left|N_{j}^{B}\right| \geqslant q_{j}$. The class of quota rules, which we denote $\mathcal{Q R}$, was introduced by Dietrich and List (2007b) in the framework of judgment aggregation. Adapting a result by Dietrich and List (2007b, Theorem 1) we obtain the following axiomatisation of the class of quota rules: ${ }^{3}$

Proposition 1. An aggregation procedure $F$ satisfies $\mathrm{A}, \mathrm{I}$, and $\mathrm{M}^{\mathrm{I}}$ on the full domain $\mathcal{D}^{\mathcal{N}}$ if and only if it is a quota rule.

A quota rule is called uniform if the quota is the same for all issues. By adding the axiom of issueneutrality to Proposition 1 we immediately get an axiomatisation of this class.

A particular quota rule, which we study in detail in Section 6.2, is the majority rule. This rule, in case the number of individuals is odd, is the uniform quota rule with quota $q=\frac{n+1}{2}$. It is interesting to link these results with May's Theorem (1952) on the axiomatic characterisation of the majority rule in voting theory. We can complement his result (which deals with the case of a single issue) by adding the axioms of domain-neutrality and issue-neutrality to Proposition 1 and considering the case of multiple binary issues. Under these assumptions, by issue-neutrality the quota must be the same for all issues, and by domain-neutrality the two sets $N_{j}^{B}$ and $\mathcal{N} \backslash N_{j}^{B}$ must be treated symmetrically. Hence, the only possibility is to have a uniform quota of $\frac{n+1}{2}$.

Proposition 2. If the number of individuals is odd and $|I| \geqslant 2$, an aggregation procedure $F$ satisfies $\mathrm{A}$, $\mathrm{N}^{\mathcal{I}}, \mathrm{N}^{\mathcal{D}}, \mathrm{I}$ and $\mathrm{M}^{\mathrm{I}}$ on the full domain $\mathcal{D}^{\mathcal{N}}$ if and only if it is the majority rule.

\section{Classes of Aggregation Procedures}

In this section we introduce two definitions for classes of aggregation procedures on binary combinatorial domains. Given a restriction on the propositional language in which integrity constraints can be expressed, we first define the class of procedures that are collectively rational with respect to all integrity constraints in the given restricted language. On the other hand, we consider a list of classical axiomatic properties and define the class of procedures that satisfy such axioms on domains defined by formulas in the given language. We study the properties of these two definitions, preparing the ground for characterisation results that will establish a strong link between requirements of collective rationality and classical axiomatic properties.

\subsection{Collective Rationality and Axiomatic Properties}

Recall that a binary aggregation problem is given by a set of agents $\mathcal{N}$ having to take a decision on which combination of binary issues in $\mathcal{I}$ to choose. Depending on the situation at hand, a subset of such combinations is designated as the set of rational choices, and this is specified by means of a propositional formula in the language $\mathcal{L}_{P S}$ associated with $\mathcal{I}$. We call language any subset $\mathcal{L}$ of $\mathcal{L}_{P S}$. Examples include the set of atoms $P S$, or the set of formulas of a given size, as well as more classical fragments obtained by restricting the set of connectives that can be employed in the construction of formulas, like the set of clauses obtained from the set of literals using only disjunctions. In Section 2.3 we called an aggregation procedure collectively rational with respect to a formula IC $\in \mathcal{L}_{P S}$ if the outcome on every rational

\footnotetext{
${ }^{3}$ Detailed proofs of Proposition 1 and Proposition 2 can be found in our previous work (Grandi, 2012a).
} 
profile satisfies the same integrity constraint IC. We now extend this definition to collectively rational procedures with respect to a given language $\mathcal{L}$ :

Definition 5. Given a language $\mathcal{L} \subseteq \mathcal{L}_{P S}$, call $\mathcal{C} \mathcal{R}[\mathcal{L}]$ the class of aggregation procedures that lift all $\mathrm{IC} \in \mathcal{L}:$

$$
\mathcal{C} \mathcal{R}[\mathcal{L}]:=\left\{F: \mathcal{D}^{\mathcal{N}} \rightarrow \mathcal{D} \mid \mathcal{N} \text { is finite and } F \text { is } C R \text { for all } \mathrm{IC} \in \mathcal{L}\right\}
$$

Note that in this definition we do not fix the number of individuals, making $\mathcal{I}$ the only parameter that is fixed in advance. This choice is arguably a natural one, as a decision problem is usually defined before specifying the number of individuals that are going to take part in the decision process. However, its appeal does not only reside in its practical use; rather it is a mathematical assumption that allows us to gain more clarity in some of the results that follow. ${ }^{4}$

The next step is to introduce notation for defining classes of aggregation procedures in terms of classical axioms like the ones we listed in Section 2.4. Recall from Section 2.4 that an axiom may be satisfied on a specific subdomain of interest $\mathcal{X}$, but not on the full domain $\mathcal{D}^{\mathcal{N}}$. Here, we are interested in domains defined by means of integrity constraints (i.e., propositional formulas), as this is interpreted as the domain of rational ballots. We therefore need some notation to identify procedures that satisfy an axiom on the subdomain $\operatorname{Mod}(\mathrm{IC})^{\mathcal{N}}$ induced by a given integrity constraint IC. We give the following definition:

Definition 6. An aggregation procedure $F$ satisfies a set of axioms $\mathrm{AX}$ wrt. a language $\mathcal{L} \subseteq \mathcal{L}_{P S}$, if $F$ satisfies the axioms in $\mathrm{AX}$ on $\operatorname{Mod}(\mathrm{IC})^{\mathcal{N}}$ for all constraints $\mathrm{IC} \in \mathcal{L}$. This defines the following class:

$$
\mathcal{F}_{\mathcal{L}}[\mathrm{AX}]:=\left\{F: \mathcal{D}^{\mathcal{N}} \rightarrow \mathcal{D} \mid \mathcal{N} \text { is finite and } F \text { satisfies } \mathrm{AX} \text { on } \operatorname{Mod}(\mathrm{IC})^{\mathcal{N}} \text { for all } \mathrm{IC} \in \mathcal{L}\right\}
$$

In particular, $\mathcal{F}=\left\{F: \mathcal{D}^{\mathcal{N}} \rightarrow \mathcal{D} \mid \mathcal{N}\right.$ is finite $\}$ is the class of all aggregation procedures for a given set of issues $\mathcal{I}$. In the sequel we shall omit mentioning explicitly that $\mathcal{N}$ is finite, keeping it as a general underlying assumption.

\subsection{Languages for Integrity Constraints}

In this section we study the behaviour of the classes defined in the previous section with respect to set-theoretic and logical operations performed on the languages and on the axioms. In particular, we give a definition of language for integrity constraints that is specific to the study of collectively rational procedures.

Let $\mathcal{L}$ be a language. Define $\mathcal{L}^{\wedge}$ to be the closure of $\mathcal{L}$ under conjunction, i.e., the set of finite conjunctions of formulas in $\mathcal{L}$. We now prove that the class of collectively rational procedures is invariant under closing the language under conjunction, i.e., that the set of collectively rational procedures for $\mathcal{L}$ and for $\mathcal{L}^{\wedge}$ coincide:

Lemma 3. $\mathcal{C} \mathcal{R}\left[\mathcal{L}^{\wedge}\right]=\mathcal{C} \mathcal{R}[\mathcal{L}]$ for all $\mathcal{L} \subseteq \mathcal{L}_{P S}$.

Proof. $\mathcal{C} R\left[\mathcal{L}^{\wedge}\right]$ is clearly included in $\mathcal{C R}[\mathcal{L}]$, since $\mathcal{L} \subseteq \mathcal{L}^{\wedge} .{ }^{5}$ It is then straightforward to observe that if an aggregation procedure $F$ lifts every constraint in $\mathcal{L}$, then it lifts any conjunction of formulas in $\mathcal{L}$.

This lemma entails that different languages for integrity constraints can define the same class of CR procedures. For instance, we have that the language of cubes (conjunctions of literals) generates the same class as the language of literals, i.e., $\mathcal{C R}[$ cubes $]=\mathcal{C} \mathcal{R}[$ literals $]$, since the former is obtained from the latter by closing it under conjunction. Another interesting fact is that procedures that are CR with respect to clauses (disjunctions of literals) are $\mathrm{CR}$ with respect to any integrity constraint in $\mathcal{L}_{P S}$, i.e.,

\footnotetext{
${ }^{4}$ Many of our results, e.g. Theorems 10 and Corollary 20, still hold if we fix the number of individuals in Definition 5, as shown in our previous work (Grandi and Endriss, 2010).

${ }^{5}$ This fact will be later formalised in Lemma 7.
} 
$\mathcal{C R}[$ clauses $]=\mathcal{C} \mathcal{R}\left[\mathcal{L}_{P S}\right]$. This holds because every propositional formula is equivalent to a formula in conjunctive normal form $(\mathrm{CNF})$, where it is expressed precisely as a conjunction of clauses.

We have just proven that the class $\mathcal{C} \mathcal{R}[\mathcal{L}]$ is invariant under closing the language under conjunction. Another such property is the closure under logical equivalence. Recall that two formulas are logically equivalent when they share the same set of models. Let us indicate with $\mathcal{L} \equiv$ the set of formulas in $\mathcal{L}_{P S}$ that are equivalent to a formula in $\mathcal{L}$. It is important to stress the fact that we consider logical equivalence inside the language $\mathcal{L}_{P S}$, not allowing the use of additional propositional variables. We have the following lemma:

Lemma 4. $\mathcal{C} \mathcal{R}\left[\mathcal{L}^{\equiv}\right]=\mathcal{C} \mathcal{R}[\mathcal{L}]$ for all $\mathcal{L} \subseteq \mathcal{L}_{P S}$.

The proof of the lemma is straightforward from our definitions. It is sufficient to observe that an equivalent formulation of our definition of collective rationality can be given by substituting formulas with the set of rational ballots given by their models. Two formulas that are logically equivalent have the same set of models, giving rise to the same requirement of collective rationality. ${ }^{6}$

Bringing together the results of Lemmas 3 and of Lemma 4, we can now give the following definition:

Definition 7. A language for integrity constraints $\mathcal{L}$ is a subset of $\mathcal{L}_{P S}$ that is closed under conjunction and logical equivalence.

In the following sections we characterise languages for integrity constraints by means of syntactic properties, without mentioning explicitly the closure under conjunction and logical equivalence. For instance, the language of 2-clauses (i.e., disjunctions of size at most two) denotes the language of formulas that are equivalent to a conjunction of clauses of size at most two. ${ }^{7}$ The language of literals and that of cubes coincide, as well as the language of clauses and the full language $\mathcal{L}_{P S}$, as we have previously remarked.

Tautologies and contradictions play a special role in languages for integrity constraints. First, observe that if a language $\mathcal{L}$ includes a tautology (or a contradiction, respectively), then by closure under logical equivalence $\mathcal{L}$ contains all tautologies (all contradictions, respectively). Thus, we indicate with $\top \in \mathcal{L}$ the fact that $\mathcal{L}$ contains all tautologies, and with $\perp \in \mathcal{L}$ the fact that $\mathcal{L}$ contains all contradictions. Second, not all languages for integrity constraints include both tautologies and contradictions, or either of them. For instance, the language of literals includes the contradiction $p \wedge \neg p$ but it does not contain any tautology. On the other hand, the language of positive clauses, consisting of clauses in which all literals occur positively, does not include either tautologies or contradictions.

Nevertheless, it is easy to see that collective rationality with respect to tautologies and contradictions corresponds to a vacuous requirement: In the first case, the outcome of a procedure will always satisfy a tautology, and in the second case the set of rational ballots is empty. These remarks constitute a proof of the following lemma.

Lemma 5. $\mathcal{C} \mathcal{R}[\mathcal{L} \cup\{\top\}]=\mathcal{C} \mathcal{R}[\mathcal{L} \cup\{\perp\}]=\mathcal{C} \mathcal{R}[\mathcal{L}]$ for all $\mathcal{L} \subseteq \mathcal{L}_{P S}$.

We now move to answering the question of whether the operations that we have included in Definition 7 are all the operations that we can perform on $\mathcal{L}$ leaving the set $\mathcal{C R}[\mathcal{L}]$ invariant. The following result provides a positive answer to this question, provided that a language include tautologies and contradictions:

Lemma 6. Given two languages for integrity constraints $\mathcal{L}_{1}$ and $\mathcal{L}_{2}$ containing $\top$ and $\perp$, if it is the case that $\mathcal{L}_{1} \neq \mathcal{L}_{2}$, then $\mathcal{C R}\left[\mathcal{L}_{1}\right] \neq \mathcal{C} \mathcal{R}\left[\mathcal{L}_{2}\right]$.

Proof. As the two languages both contain tautologies and contradictions, they must differ on a contingent formula $\varphi$. Without loss of generality we can consider a formula $\varphi \in \mathcal{L}_{2}$ such that $\varphi \notin \mathcal{L}_{1}$. We want to prove that there exists an aggregation procedure $F \in \mathcal{C R}\left[\mathcal{L}_{1}\right]$ that is not $\mathrm{CR}$ with respect to $\varphi$. This in turn implies that $F$ is not in $\mathcal{C R}\left[\mathcal{L}_{2}\right]$, and that the two classes $\mathcal{C R}\left[\mathcal{L}_{1}\right]$ and $\mathcal{C R}\left[\mathcal{L}_{2}\right]$ are different.

\footnotetext{
${ }^{6}$ This is the standard approach in the literature on binary aggregation (see, e.g., Dokow and Holzman, 2010a). Our choice of using formulas rather than sets is motivated by the compactness of this representation and by the possibility of using syntax to classify rationality assumptions.

${ }^{7}$ The language of 2 -clauses can be equivalently defined by closing the set of 2-CNF under logical equivalence.
} 
Let $|\mathcal{N}|=n$ where $n=|\operatorname{Mod}(\varphi)|$ and let $F$ be a procedure in $\mathcal{C} \mathcal{R}\left[\mathcal{L}_{1}\right]$ defined for $\mathcal{N}^{8}$. We claim that it is possible to modify the behaviour of $F$ on a single profile $\boldsymbol{B}$ in order to create another procedure $F^{\prime}$ that is still $\mathrm{CR}$ with respect to $\mathcal{L}_{1}$ but sends the profile $\boldsymbol{B}$ of $\varphi$-rational ballots to an outcome that does not satisfy $\varphi$. To do so it is sufficient to find a profile $\boldsymbol{B}=\left(B_{1}, \ldots, B_{n}\right)$ of models of $\varphi$ and a ballot $B_{c}$ outside $\operatorname{Mod}(\varphi)$ such that whenever each of $B_{1}, \ldots, B_{n}$ satisfies any formula $\psi \in \mathcal{L}_{1}$ then also $B_{c} \models \psi$. If we can find such a $\boldsymbol{B}$ and $B_{c}$, then by setting $F^{\prime}(\boldsymbol{B})=B_{c}$ and $F^{\prime}\left(\boldsymbol{B}^{\prime}\right)=F\left(\boldsymbol{B}^{\prime}\right)$ for all remaining $\boldsymbol{B}^{\prime} \neq \boldsymbol{B}$ we obtain an aggregation procedure that is in $\mathcal{C R}\left[\mathcal{L}_{1}\right]$ but not in $\mathcal{C R}\left[\mathcal{L}_{2}\right]$.

Suppose for the sake of contradiction that such a profile does not exist, i.e., that for every choice of $n$ ballots in $\operatorname{Mod}(\varphi)$ and ballot $B_{c}$ outside $\operatorname{Mod}(\varphi)$, there is a formula $\psi \in \mathcal{L}_{1}$ that separates them: for all $i$ we have that $B_{i} \mid=\psi$ but $B_{c} \not \models \psi$. Note that $|\operatorname{Mod}(\varphi)|=n$, as well as the size of the profile $\boldsymbol{B}$ we are looking for. This entails that we can construct a profile $\boldsymbol{B}_{\varphi}$ that contains all distinct models of $\varphi$, and that for every $B_{c} \not \models \varphi$ there is a formula in $\mathcal{L}_{1}$, that we shall call $\psi_{c}$, that separates the set $\operatorname{Mod}(\varphi)$ from $B_{c}$.

We have assumed that $\varphi \notin \mathcal{L}_{1}$, i.e., $\varphi$ is not equivalent to a conjunction of formulas in $\mathcal{L}_{1}$. Let us now consider the conjunction $\bigwedge \psi_{c}$ for $B_{c} \not \models \varphi$. We claim that $\varphi \equiv \bigwedge \psi_{c}$, in contradiction with our assumption, since all $\psi_{c}$ are in $\mathcal{L}_{1}$. By construction we know that $\operatorname{Mod}(\varphi) \subseteq \operatorname{Mod}\left(\bigwedge \psi_{c}\right)$, as all $\operatorname{models}$ of $\varphi$ are individual ballots in the profile $\boldsymbol{B}_{\varphi}$. We need to prove the other inclusion. Assume for the sake of contradiction that there exists a $B_{*}$ in $\operatorname{Mod}\left(\bigwedge \psi_{c}\right) \backslash \operatorname{Mod}(\varphi)$. By construction, there exists a formula $\psi_{*} \in \mathcal{L}_{1}$ that separates $B_{*}$ from $\operatorname{Mod}(\varphi)$, and this formula is included in $\Lambda \psi_{c}$. But by construction $B_{*} \not \models \psi_{*}$, therefore it cannot be included in $\operatorname{Mod}\left(\bigwedge \psi_{c}\right)$.

The proof is now concluded: it is possible to modify $F$ on the profile $\boldsymbol{B}$ that contains all models of $\varphi$ to output a ballot that is not a model of $\varphi$ but respects all integrity constraints in $\mathcal{L}_{1}$. That is, $F^{\prime}$ so defined is in $\mathcal{C R}\left[\mathcal{L}_{1}\right]$ but not in $\mathcal{C} \mathcal{R}[\varphi]$.

We conclude this section by establishing some easy properties of $\mathcal{C} \mathcal{R}[\mathcal{L}]$ and of $\mathcal{F}_{\mathcal{L}}[\mathrm{AX}]$ that shall be useful in the next sections. Let $\mathcal{L}_{1}$ and $\mathcal{L}_{2}$ be languages for integrity constraints.

Lemma 7. The following facts hold:

(i) If $\mathcal{L}_{1} \subseteq \mathcal{L}_{2}$, then $\mathcal{C} \mathcal{R}\left[\mathcal{L}_{1}\right] \supseteq \mathcal{C R}\left[\mathcal{L}_{2}\right]$;

(ii) $\mathcal{C R}\left[\mathcal{L}_{1} \cup \mathcal{L}_{2}\right]=\mathcal{C} \mathcal{R}\left[\mathcal{L}_{1}\right] \cap \mathcal{C R}\left[\mathcal{L}_{2}\right]$ for all $\mathcal{L}_{1}, \mathcal{L}_{2} \subseteq \mathcal{L}_{P S}$.

The proof is straightforward from our definitions. Lemma 7 still holds if $\mathcal{L}_{1}$ and $\mathcal{L}_{2}$ are arbitrary sets of propositional formulas (e.g., in the proof of Lemma 3 we are implicitly making use of point $(i)$ of Lemma 7). Similar properties can be proved for classes of procedures defined in terms of axioms. We write $\mathcal{F}[\mathrm{AX}]$ as a shorthand for $\mathcal{F}_{\{T\}}[\mathrm{AX}]$, the class of procedures that satisfy the axioms in AX over the full domain $\mathcal{D}$. It is easy to see that the following lemma holds:

Lemma 8. The following facts hold:

(i) if $\mathcal{L}_{1} \subseteq \mathcal{L}_{2}$ then $\mathcal{F}_{\mathcal{L}_{1}}[\mathrm{AX}] \supseteq \mathcal{F}_{\mathcal{L}_{2}}[\mathrm{AX}]$;

(ii) in particular, if $\top \in \mathcal{L}$, then $\mathcal{F}[\mathrm{AX}] \supseteq \mathcal{F}_{\mathcal{L}}[\mathrm{AX}]$;

(iii) $\mathcal{F}_{\mathcal{L}}\left[\mathrm{AX}_{1}, \mathrm{AX}_{2}\right]=\mathcal{F}_{\mathcal{L}}\left[\mathrm{AX}_{1}\right] \cap \mathcal{F}_{\mathcal{L}}\left[\mathrm{AX}_{2}\right]$.

Observe that for most axioms an additional fact holds: if the axiomatic property AX is satisfied on the full domain $\mathcal{D}$, then $\mathrm{AX}$ is also satisfied on every subdomain of $\mathcal{D}$. This is true in particular for all the axioms we considered in Section 2.4. Thus, for most axioms AX the following additional property holds: $\mathcal{F}[\mathrm{AX}] \subseteq \mathcal{F}_{\mathcal{L}}[\mathrm{AX}]$ for all $\mathcal{L} \subseteq \mathcal{L}_{P S}$. By $($ ii $)$ of Lemma 8 this entails that if $T \in \mathcal{L}$ then $\mathcal{F}[\mathrm{AX}]=\mathcal{F}_{\mathcal{L}}[\mathrm{AX}]$ for all $\mathcal{L} \subseteq \mathcal{L}_{P S}$.. This observation will allow us in Section 5 to obtain stronger characterisation results by dropping the subscript in the definition of the class $\mathcal{F}_{\mathcal{L}}[\mathrm{AX}]$.

\footnotetext{
${ }^{8}$ In Section 4 we prove that $\mathcal{C} \mathcal{R}[\mathcal{L}]$ can never be empty for any language $\mathcal{L}$ and any set of agents $\mathcal{N}$ (see Theorem 16). For the sake of this proof it is sufficient to consider a dictatorship of the first individual.
} 


\subsection{From Aggregation Procedures to Integrity Constraints and Back}

In the first part of Section 3 we have associated with any language for integrity constraints $\mathcal{L}$ a class of aggregation procedures that are collectively rational with respect to all formulas in $\mathcal{L}$. Once a set of issues $\mathcal{I}$ is fixed, $\mathcal{C R}[-]$ can therefore be viewed as an operator from the set of languages for integrity constraints (i.e., subsets of $\mathcal{L}_{P S}$ closed under conjunction and logical equivalence and containing $T$ and $\perp$ ) to subsets of the class $\mathcal{F}$ of all aggregation procedures for $\mathcal{I}$. In this section we introduce an inverse operation, that we shall call $\mathcal{L F}[-]$, which, given a class of procedures, outputs the set of integrity constraints that are lifted by all procedures in that class. As we will see, $\mathcal{L F}$ is the left inverse of $\mathcal{C R}$, but on the other hand the two operators do not commute.

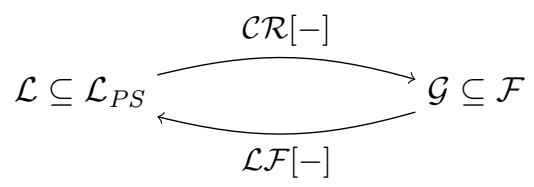

Figure 1: The operators $\mathcal{C R}[-]$ and $\mathcal{L F}[-]$.

Definition 8. Given a class of aggregation procedures $\mathcal{G} \subseteq \mathcal{F}$, call $\mathcal{L} \mathcal{F}[\mathcal{G}]$ the set of integrity constraints that are lifted by all $F \in \mathcal{G}$ :

$$
\mathcal{L} \mathcal{F}[\mathcal{G}]:=\left\{\varphi \in \mathcal{L}_{P S} \mid F \text { is } C R \text { wrt. } \varphi \text { for all } F \in \mathcal{G}\right\}
$$

$\mathcal{L F}[\mathcal{G}]$ is the intersection of all $\mathcal{L F}[\{F\}]$ for $F \in \mathcal{G}$. We now prove the following:

Proposition 9. Let $\mathcal{I}$ be a set of issues, $\mathcal{L}$ a language for integrity constraints containing $\top$ and $\perp$, and $\mathcal{G} \subseteq \mathcal{F}$ a class of aggregation procedures on $\mathcal{I}$. Then the following facts are true:

(i) $\mathcal{L} \mathcal{F}[\mathcal{C R}[\mathcal{L}]]=\mathcal{L}$

(ii) $\mathcal{C R}[\mathcal{L} \mathcal{F}[\mathcal{G}]] \supseteq \mathcal{G}$ and this inclusion is strict for some classes.

Proof. (i) We start by proving that $\mathcal{L F}$ is a left inverse of $\mathcal{C R}$. A direct consequence of our definitions is that $\mathcal{L} \subseteq \mathcal{L F}[\mathcal{C R}[\mathcal{L}]]$, and we now prove the other inclusion. We want to show that if an integrity constraint $\varphi$ is lifted by all procedures that are CR with respect to $\mathcal{L}$, then $\varphi$ belongs to $\mathcal{L}$. This is a straightforward consequence of Lemma 6 . Assume for the sake of contradiction that $\varphi \notin \mathcal{L}$. By Lemma 6, there exists a procedure $F$ which is collectively rational for $\mathcal{L}$ but not for $\varphi$, in contradiction to our assumption that all procedures in $\mathcal{C R}[\mathcal{L}]$ are $\mathrm{CR}$ with respect to $\varphi$. Therefore $\varphi$ is in $\mathcal{L}$.

(ii) It is straightforward from our definitions that $\mathcal{C R}[\mathcal{L F}[\mathcal{G}]] \supseteq \mathcal{G}$. Recall that a generalised dictatorship is a procedure that copies the ballot of a possibly different individual in every profile. It can be easily observed that such a procedure is collectively rational for every integrity constraint, and in Section 2.2 we will give a formal proof of this fact (cf. Theorem 16). Let us therefore consider a class of procedures $\mathcal{G}$ not containing any generalised dictatorship. In view of our previous observation we know that all generalised dictatorships are contained in $\mathcal{C R}[\mathcal{L F}[\mathcal{G}]]$, as they are collectively rational for any integrity constraint. As we assumed that $\mathcal{G}$ does not contain any generalised dictatorship, we infer that $\mathcal{C R}[\mathcal{L F}[\mathcal{G}]] \supsetneq \mathcal{G}$.

\section{Characterisation Results for Propositional Languages}

Once a language for integrity constraints is fixed, e.g., by means of a syntactic restriction on the integrity constraints, we may be interested in the problem of how to guarantee collective rationality with respect to all the integrity constraints that can be expressed in the given language. The aim of this section is 
to explore the relationship between the two definitions of classes of aggregation procedures introduced in Section 3: collectively rational procedures on one side, and procedures defined by axiomatic requirements on the other. In particular, we look for results of the following form:

$$
\mathcal{C R}[\mathcal{L}]=\mathcal{F}_{\mathcal{L}}[\mathrm{AX}],
$$

for languages $\mathcal{L}$ and axioms AX. We call such findings characterisation results: they provide necessary and sufficient axiomatic conditions for an aggregation procedure to be collectively rational with respect to a language for integrity constraints. Given the importance of collective rationality in many classical studies of aggregation and for a number of practical applications, such results are central to our study.

The focus of this section is on languages: We provide complete characterisation for some basic classes of languages defined in a syntactic fashion, proving the correspondence with some of the main classical axioms from the literature on SCT. We shift the focus to axioms in Section 5.

Definitions of all the axiomatic properties we refer to in this section can be found in Section 2.4. Axioms will be denoted with the capital letter associated with them, e.g., we will write $\mathrm{U}$ for unanimity and I for independence.

\subsection{Characterisation Results}

Recall that a procedure is unanimous if it shares the view of the individuals in case they all agree, either all accepting or rejecting a certain issue. The first characterisation result shows that the set of aggregation procedures that lift all rationality constraints expressible in terms of literals is precisely the class of unanimous procedures:

Theorem 10. $\mathcal{C} \mathcal{R}[$ literals $]=\mathcal{F}_{\text {literals }}[\mathrm{U}]$.

Proof. The first direction $(\supseteq)$ is easy: If $X:=\operatorname{Mod}(\ell)$ is a domain defined by a literal $\ell$, then every individual ballot must agree with it, either positively or negatively depending on its sign. This entails, by unanimity, that the collective outcome agrees with the individual ballots. Thus, $F$ is collectively rational for $\ell$.

For the other direction $(\subseteq)$, suppose that $F \in \mathcal{C} \mathcal{R}$ [literals]. Fix an issue $j \in \mathcal{I}$. Pick a profile $\boldsymbol{B} \in \mathcal{D}^{n}$ such that $b_{i, j}=1$ (or 0 ) for all $i \in \mathcal{N}$. That is, $\boldsymbol{B} \in \operatorname{Mod}\left(p_{j}\right)^{\mathcal{N}}$ (or $\neg p_{j}$, respectively). Since $F$ is collectively rational for every literal, including $p_{j}$ and $\neg p_{j}$, it must be the case that $F(\boldsymbol{B})_{j}=1$ (or 0 , respectively), proving unanimity of the aggregator.

As remarked in Section 3, the language generated from literals is the same as the language of cubes, i.e., finite conjunctions of literals. We can therefore state the following corollary:

Corollary 11. $\mathcal{C} R[$ cubes $]=\mathcal{F}_{\text {cubes }}[\mathrm{U}]$.

An equivalence is a bi-implication of literals where the literals are both positive (or both negative, which amounts to the same thing). Call $\mathcal{L}_{\leftrightarrow}$ the language for integrity constraints generated by equivalences, i.e., the set $\left\{p_{j} \leftrightarrow p_{k} \mid p_{j}, p_{k} \in P S\right\}$ closed under conjunctions and logical equivalence. This language allows us to characterise issue-neutral aggregators, i.e., procedures that treat distinct issues in the same way:

Theorem 12. $\mathcal{C R}\left[\mathcal{L}_{\leftrightarrow}\right]=\mathcal{F}_{\mathcal{L}_{\leftrightarrow}}\left[\mathrm{N}^{\mathcal{I}}\right]$.

Proof. To prove the first inclusion (), pick an equivalence $p_{j} \leftrightarrow p_{k}$. This defines a domain in which issues $j$ and $k$ share the same pattern of acceptance/rejection, and since the procedure is neutral over issues, we get $F(\boldsymbol{B})_{j}=F(\boldsymbol{B})_{k}$. Therefore, the constraint given by the initial equivalence is lifted.

For the other direction $(\subseteq)$, suppose that a profile $\boldsymbol{B}$ is such that $b_{i, j}=b_{i, k}$ for every $i \in \mathcal{N}$. This implies that $\boldsymbol{B} \in \operatorname{Mod}\left(p_{j} \leftrightarrow p_{k}\right)^{\mathcal{N}}$, and since $F$ is in $\mathcal{C} \mathcal{R}\left[\mathcal{L}_{\leftrightarrow}\right], F(\boldsymbol{B})_{j}$ must be equal to $F(\boldsymbol{B})_{k}$. This holds for every such $\boldsymbol{B}$, proving that $F$ is neutral over issues. 
With an analogous proof we can obtain a characterisation result involving the axiom of domain-neutrality. Recall that a procedure is domain-neutral if it symmetric with respect to any two issues. An XOR formula is a bi-implication of one negative and one positive literal. Call $\mathcal{L}_{X O R}$ the language for integrity constraints generated from $\left\{p_{j} \leftrightarrow \neg p_{k} \mid p_{j}, p_{k} \in P S\right\}$.

Theorem 13. $\mathcal{C} \mathcal{R}\left[\mathcal{L}_{X O R}\right]=\mathcal{F}_{\mathcal{L}_{X O R}}\left[\mathrm{~N}^{\mathcal{D}}\right]$.

Proof. The first inclusion $(\supseteq)$ is straightforward: When every individual ballot in a profile satisfies the same XOR formula, then this means that there are two issues the behaviour of which is symmetrical. By domain-neutrality, the outcome of the aggregation is also symmetrical, and therefore the constraint is lifted.

To prove the remaining inclusion $(\subseteq)$, suppose that a profile $\boldsymbol{B}$ is such that $b_{i, j}=1-b_{i, k}$ for every $i \in \mathcal{N}$. This implies that $\boldsymbol{B} \in \operatorname{Mod}\left(p_{j} \leftrightarrow \neg p_{k}\right)^{\mathcal{N}}$. As before, since $F$ is in $\mathcal{C} \mathcal{R}\left[\mathcal{L}_{X O R}\right]$, it must be the case that $F(\boldsymbol{B})_{j}=1-F(\boldsymbol{B})_{k}$ and $F$ is domain-neutral.

Consider now the language $\mathcal{L}_{\rightarrow}^{+}$of positive implications, generated from formulas of the form $p_{j} \rightarrow p_{k}$, or, equivalently, $\neg p_{j} \rightarrow \neg p_{k}$, for $p_{j}, p_{k} \in P S$. Since $\mathcal{L}_{\rightarrow}^{+} \supseteq \mathcal{L}_{\leftrightarrow}$, we know that $\mathcal{C R}\left[\mathcal{L}_{\rightarrow}^{+}\right] \subseteq \mathcal{C R}\left[\mathcal{L}_{\leftrightarrow}\right]=\mathcal{F}_{\mathcal{L}_{\leftrightarrow}}\left[\mathrm{N}^{\mathcal{I}}\right]$. Therefore, a characterisation of the language of positive implications must involve the axiom of neutrality in combination with others. The right combination is the following:

Theorem 14. $\mathcal{C} \mathcal{R}\left[\mathcal{L}_{\rightarrow}^{+}\right]=\mathcal{F}_{\mathcal{L}_{\stackrel{+}{+}}^{+}}\left[\mathrm{N}^{\mathcal{I}}, \mathrm{M}^{\mathrm{N}}\right]$.

Proof. ( $\supseteq$ ) Let us first consider the case of individual ballots all satisfying a certain positive implication $p_{j} \rightarrow p_{k}$. We want to prove that $F$ lifts this integrity constraint. We note that if an individual accepts issue $j$ then she also accepts issue $k$. Therefore, the first part of the antecedent forming the axiom of Nmonotonicity is satisfied. We now have to consider two cases: if for all $i \in \mathcal{N}$ we have that $b_{i, j}=b_{i, k}=1$, then by issue-neutrality we have that $F(\boldsymbol{B})_{j}=F(\boldsymbol{B})_{k}$. The constraint is therefore satisfied, as the only way to falsify it is by accepting $j$ and rejecting $k$. If on the other hand there is an individual $i$ such that $b_{i, j}=0$ while $b_{i, k}=1$, then $\boldsymbol{B}$ fully satisfies the antecedent of $\mathrm{M}^{\mathrm{N}}$ and therefore the constraint will be lifted.

For the remaining inclusion $(\subseteq)$, suppose that a profile $\boldsymbol{B}$ is such that whenever $b_{i, j}=1$ then $b_{i, k}=1$ for every $i \in \mathcal{N}$. This implies that $\boldsymbol{B} \in \operatorname{Mod}\left(p_{j} \rightarrow p_{k}\right)^{\mathcal{N}}$. Since we assumed $F$ to be in $\mathcal{C} \mathcal{R}\left[\mathcal{L}_{\rightarrow}^{+}\right]$, $F(\boldsymbol{B})_{j}=1$ entails $F(\boldsymbol{B})_{k}=1$, for the initial constraint has to be lifted. Therefore $F$ is N-monotonic. We have already remarked that, due to Proposition 12, all procedures in $\mathcal{C R}\left[\mathcal{L}_{\rightarrow}^{+}\right]$are also issue-neutral.

This last result seems to suggest that a characterisation of the language of negative implications (i.e., when exactly one of the two literals is negative) might be proved by considering the axiom of domainneutrality combined with N-monotonicity. Unfortunately, in the absence of a suitable richness condition on the profile this characterisation does not hold. A partial characterisation result for this class, i.e., a list of sufficient axiomatic conditions for collective rationality with respect to negative implications, involves the axiom of independence, and can be found in our previous work (Grandi, 2012a).

We conclude this section by characterising the classes of collectively rational procedures for languages at the extremes of the spectrum: the full language $\mathcal{L}_{P S}$, the language of tautologies, and that of contradictions. For the last two classes the characterisation is straightforward. Recall that $\mathcal{F}=\left\{F: \mathcal{D}^{\mathcal{N}} \rightarrow \mathcal{D}\right\}$ is the class of all aggregation procedures (for fixed $\mathcal{I}$ ). We have already stated in Lemma 5 that tautologies and contradictions are vacuous requirements for what concerns collective rationality, and here we use these arguments to give a characterisation result for this trivial class of formulas. Let $\{\top\}$ be the language of all tautologies, and $\{\perp\}$ be the language of all contradictions:

Proposition 15. $\mathcal{C R}[\{\top\}]=\mathcal{C} \mathcal{R}[\{\perp\}]=\mathcal{F}$.

If on the other hand we turn to study the class of procedures that lift any integrity constraint in $\mathcal{L}_{P S}$ we discover an interesting class of procedures. Let us give the following definition, which generalises the notion of dictatorship: ${ }^{9}$

\footnotetext{
${ }^{9}$ An analogous definition was given by Cariani et al. (2008) in the context of judgment aggregation, under the name of rolling dictatorships.
} 
Definition 9. An aggregation procedure $F: \mathcal{D}^{\mathcal{N}} \rightarrow \mathcal{D}$ is a generalised dictatorship, if there exists a map $g: \mathcal{D}^{\mathcal{N}} \rightarrow \mathcal{N}$ such that $F(\boldsymbol{B})=B_{g(\boldsymbol{B})}$ for every $\boldsymbol{B} \in \mathcal{D}^{\mathcal{N}}$.

That is, a generalised dictatorship copies the ballot of a (possibly different) individual in every profile. Call this class GDIC. This class fully characterises the class of collectively rational aggregators for the full propositional language $\mathcal{L}_{P S}$ :

Theorem 16. $\mathcal{C} \mathcal{R}\left[\mathcal{L}_{P S}\right]=$ GDIC.

Proof. Clearly, every generalised dictatorship lifts any arbitrary integrity constraint IC $\in \mathcal{L}_{P S}$. To prove the other direction, suppose that $F \notin \mathcal{F}\left[\right.$ GDIC]. Hence, there exists a profile $\boldsymbol{B} \in \mathcal{D}^{\mathcal{N}}$ such that $F(\boldsymbol{B}) \neq B_{i}$ for all $i \in \mathcal{N}$. This means that for every $i$ there exists an issue $j_{i}$ such that $F(\boldsymbol{B})_{j_{i}} \neq b_{i, j_{i}}$. We now want to build a propositional formula that is satisfied by all individuals and not by the collective outcome, proving that $F$ is not $\mathrm{CR}$ with respect to the full propositional language. Define a literal $\ell_{j_{i}}$ to be equal to $p_{j_{i}}$ if $b_{i, j_{i}}=1$, and to $\neg p_{j_{i}}$ otherwise. Consider as integrity constraint IC the following formula: $\bigvee_{i} \ell_{j_{i}}$. Clearly, $B_{i}=\mathrm{IC}$ for every $i \in \mathcal{N}$, i.e., $\boldsymbol{B}$ is a rational profile for the integrity constraint IC. But by construction, $F(\boldsymbol{B}) \not \models \mathrm{IC}$, as $F(\boldsymbol{B})$ differs from the individual ballots on all literals in IC. Therefore, $F$ is not collectively rational for IC and does not belong to the class $\mathcal{C} R\left[\mathcal{L}_{P S}\right]$.

As a concluding remark, recall that the language generated by clauses coincides with the full propositional language, as every propositional formula is equivalent to a conjunction of clauses by taking its conjunctive normal form. We therefore obtain the following:

Corollary 17. $\mathcal{C R}[$ clauses $]=$ GDIC.

We analyse restricted languages of clauses in Section 6 .

\subsection{How to Combine Characterisation Results}

Most of the characterisation results presented thus far characterise a class of procedures determined by a single axiom and by a uniform description of the language. We now briefly explain to what extent such results can be combined to allow us to make predictions regarding the collective rationality of procedures satisfying several such axioms, or in the case where the integrity constraints can be chosen from a more complex language.

Consider the case of two characterisation results $\mathcal{C R}\left[\mathcal{L}_{1}\right]=\mathcal{F}_{\mathcal{L}_{1}}\left[\mathrm{AX}_{1}\right]$ and $\mathcal{C} \mathcal{R}\left[\mathcal{L}_{2}\right]=\mathcal{F}_{\mathcal{L}_{2}}\left[\mathrm{AX}_{2}\right]$. By part (ii) of Lemma 7 and by $\mathcal{F}_{\mathcal{L}_{1} \cup \mathcal{L}_{2}}\left[\mathrm{AX}_{1}, \mathrm{AX}_{2}\right] \subseteq \mathcal{F}_{\mathcal{L}_{1}}\left[\mathrm{AX}_{1}\right] \cap \mathcal{F}_{\mathcal{L}_{2}}\left[\mathrm{AX}_{2}\right]$ we can infer that:

$$
\mathcal{F}_{\mathcal{L}_{1} \cup \mathcal{L}_{2}}\left[\mathrm{AX}_{1}, \mathrm{AX}_{2}\right] \subseteq \mathcal{C R}\left[\mathcal{L}_{1} \cup \mathcal{L}_{2}\right]
$$

(But note that the other inclusion is not always true.) This entails that if we express constraints in the language $\mathcal{L}_{1} \cup \mathcal{L}_{2}$ or in any of its sublanguages, then picking procedures from $\mathcal{F}_{\mathcal{L}_{1} \cup \mathcal{L}_{2}}\left[\mathrm{AX}_{1}, \mathrm{AX}_{2}\right]$ is a sufficient condition for collective rationality. If, instead, we start from a class of procedures satisfying axioms $\mathrm{AX}_{1}$ and $\mathrm{AX}_{2}$ on the language $\mathcal{L}_{1} \cup \mathcal{L}_{2}$ then we can infer that these procedures lift any language $\mathcal{L} \subseteq \mathcal{L}_{1} \cup \mathcal{L}_{2}$, since as we previously observed $\mathcal{F}_{\mathcal{L}_{1} \cup \mathcal{L}_{2}}\left[\mathrm{AX}_{1}, \mathrm{AX}_{2}\right]$ is included in $\mathcal{C} \mathcal{R}\left[\mathcal{L}_{1} \cup \mathcal{L}_{2}\right]$, which in turn is included in $\mathcal{C R}[\mathcal{L}]$.

\section{Characterisation Results for Classical Axioms}

In the previous section we proved several characterisation results for various simple fragments of the propositional language associated with an aggregation problem. In this section we shift our focus from syntactic descriptions of languages to axiomatic properties of aggregation procedures, having the axioms as variables when exploring the possibility for a characterisation result. We first show that for most characterisation results proved in the previous section the domain restriction given by the language $\mathcal{L}$ can be dropped, thus obtaining characterisation results for classical axioms from SCT. Then, we prove a negative result involving the axioms of anonymity, independence, and both forms of monotonicity, showing that a characterisation result cannot be proved for these axioms. 


\subsection{Characterisation of Axioms}

Consider the class $\mathcal{F}[\mathrm{AX}]$, dropping the subscript $\mathcal{L}$, as representing the class of procedures that defines an axiom. For every domain restriction, we know that $\mathcal{F}[\mathrm{AX}] \subseteq \mathcal{F}_{\mathcal{L}}[\mathrm{AX}]$. As anticipated at the end of Section 3.2, most of the characterisation results presented in the previous section can be proved without the restriction on $\mathcal{L}$ on the right-hand side, becoming therefore characterisations of classical axioms:

Corollary 18. The following equivalences hold:

(i) $\mathcal{F}[\mathrm{U}]=\mathcal{C R}[$ literals $]$.

(ii) $\mathcal{F}\left[\mathrm{N}^{\mathcal{I}}\right]=\mathcal{C} \mathcal{R}\left[\mathcal{L}_{\leftrightarrow}\right]$.

(iii) $\mathcal{F}\left[\mathrm{N}^{\mathcal{D}}\right]=\mathcal{C} \mathcal{R}\left[\mathcal{L}_{X O R}\right]$.

Proof. Refer to Theorems 10, 12 and 13. For all three classes we prove that $\mathcal{F}_{\mathcal{L}}[\mathrm{AX}]=\mathcal{F}[\mathrm{AX}]$, for the relevant axiom and language. This can be seen by observing that, in the three cases under consideration, the condition required by the axiom is a vacuous requirement outside domains defined by formulas in $\mathcal{L}$. Therefore, if a procedure satisfies an axiom on domains defined by $\mathcal{L}$ then it also satisfies the same axiom on the full domain.

\subsection{Negative Results}

Results such as the one proved in the previous section cannot be proved for other important axioms, for which it is not possible to obtain a characterisation result. In this section we prove a negative result for the axioms of independence, anonymity and both formulations of monotonicity. We first prove the following proposition. Recall that $\mathcal{L F}[\mathcal{G}]$ is the set of integrity constraints lifted by all the aggregation procedures in $\mathcal{G}$, and let $\mathcal{L}_{\top, \perp}$ be the language for integrity constraints generated by $\{\top, \perp\}$.

Proposition 19. The following hold:

$$
\mathcal{L F} \mathcal{F}[\mathrm{F}[]]=\mathcal{L} \mathcal{F}[\mathcal{F}[\mathrm{A}]]=\mathcal{L} \mathcal{F}\left[\mathcal{F}\left[\mathrm{M}^{\mathrm{I}}\right]\right]=\mathcal{L} \mathcal{F}\left[\mathcal{F}\left[\mathrm{M}^{\mathrm{N}}\right]\right]=\mathcal{L}_{\mathrm{T}, \perp}
$$

Proof. We prove this proposition by constructing for any contingent formula $\varphi$ (i.e., such that both $\varphi$ and $\neg \varphi$ are satisfiable) an independent, anonymous and monotonic procedure that is not collectively rational with respect to $\varphi$. Let $\varphi$ be such a formula, and let $B^{\star} \in \mathcal{D}$ be a ballot such that $B^{\star} \not \models \varphi$. Consider now the constant procedure $F(\boldsymbol{B})=B^{\star}$ for all profiles $\boldsymbol{B}$ : this procedure is independent, anonymous and monotonic, but it is not collectively rational with respect to $\varphi$.

An immediate corollary of this result is that it is not possible to obtain a characterisation of these axioms in terms of collective rationality:

Corollary 20. There is no language for integrity constraints $\mathcal{L} \subseteq \mathcal{L}_{P S}$ such that $\mathcal{C} \mathcal{R}[\mathcal{L}]=\mathcal{F}[\mathrm{I}]$. The same holds for $\mathcal{F}[\mathrm{A}], \mathcal{F}\left[\mathrm{M}^{\mathrm{I}}\right]$ and $\mathcal{F}\left[\mathrm{M}^{\mathrm{N}}\right]$.

Proof. By Proposition 9, in the presence of a characterisation result the set of integrity constraints lifted by a class of procedures is uniquely determined. Suppose then that $\mathcal{C R}[\mathcal{L}]=\mathcal{F}[\mathrm{AX}]$ for $\mathrm{AX} \in$ $\left\{\mathrm{I}, \mathrm{A}, \mathrm{M}^{\mathrm{I}}, \mathrm{M}^{\mathrm{N}}\right\}$ and for a certain $\mathcal{L}$. Proposition 19 forces $\mathcal{L}$ to be equal to $\mathcal{L}_{\mathrm{T}, \perp}$, but we have already proven that this class characterises the whole set of procedures $\mathcal{F}$ (cf. Proposition 15 combined with Lemma 7). Therefore, such a characterisation cannot exist.

Note that this argument can be generalised to prove that the class $\mathcal{F}_{\mathcal{L}}[\mathrm{I}]$ (and the same holds for $\mathrm{A}$, $\mathrm{M}^{\mathrm{I}}$ and $\mathrm{M}^{\mathrm{N}}$ ) cannot be characterised for any restriction given by a language $\mathcal{L}$. It is sufficient to note that the constant procedure employed in the proof of Proposition 19 is defined regardless of the domain restriction.

Our interest in these axioms does not cease here. On the contrary, the last two results showed that the classes of monotone, independent and anonymous procedures behave in the same way as the full class $\mathcal{F}$ of all aggregation procedures for what concerns collective rationality. This suggests that interesting 
characterisations can be studied inside those classes, replacing the set $\mathcal{F}$ with, e.g., the class $\mathcal{F}[\mathrm{I}]$. We are going to pursue a similar approach in the following section by focusing on the class $\mathcal{F}\left[\mathrm{A}, \mathrm{I}, \mathrm{M}^{\mathrm{I}}\right]$, i.e., the class of quota rules.

\section{Quota Rules and Languages of Clauses}

This section is devoted to a thorough exploration of the classes of collectively rational procedures for several languages of clauses inside the class of quota rules. In Section 6.1 we begin by characterising quota rules that are collectively rational with respect to positive or negative clauses, i.e., clauses in which literals are either all positive or all negative. Then in Section 6.2 we focus on arbitrary clauses of size 2, showing that this class coincides with the set of integrity constraints that are lifted by the majority rule. Finally, in Section 6.3 we provide a general result which characterises quota rules that are collectively rational with respect to an arbitrary clause of a given size.

We introduced quota rules in Section 2.5 as procedures assigning a quota $q_{j}$ to every issue $j$ so that $F(\boldsymbol{B})_{j}=1 \Leftrightarrow\left|\left\{i \mid b_{i, j}=1\right\}\right| \geqslant q_{j}$. In the same section we also proved that quota rules are axiomatised as the class of independent, anonymous and monotone procedures. If we denote with $\mathcal{Q R}$ the set of quota rules, then we can write $\mathcal{Q R}=\mathcal{F}\left[\mathrm{A}, \mathrm{I}, \mathrm{M}^{\mathrm{I}}\right]$. Recall that if $|\mathcal{N}|=n$, quotas $q_{j}$ are integers between 0 and $n+1$, the two extreme cases of $q_{j}=0$ and $q_{j}=n+1$ being the two constant rules that output, respectively, 1 and 0 .

There are several reasons why the choice of quota rules and languages of clauses constitutes an interesting combination. First, since we have proven in Section 5 that no characterisation result is possible neither for independent, nor for anonymous, nor for monotonic procedures, it is important to explore classes of collectively rational procedures inside those classes. As remarked earlier, by combining all three axioms together we obtain the set of quota rules. Second, languages of clauses are the most expressive ones, ranging from literals, to implications, to the full expressivity of $\mathcal{L}_{P S}$. By Corollary 17 we know that to obtain interesting results it is necessary to limit either the size or the shape of the clauses that build up a language for integrity constraints. In this section we concentrate on languages defined by bounding the size of clauses, and quota rules seem a perfect candidate to deal with such restrictions, as they allow us to play with quotas and constrain them with equations. Clauses of limited size are of interest in the modelling of several applications, e.g., for limiting cluster size in graph aggregation (Endriss and Grandi, 2012), or for bounding the number of premises in Horn formulas.

We will assume for the rest of this section that the number of issues is always strictly bigger than the limitation on the size of a clause. This is because in case the number of issues is smaller or equal than the bound on clauses this limitation is fictitious. For $k \geqslant 1$, we define an exact $k$-clause as a clause of length $k$, i.e., a clause in which exactly $k$ propositional symbols occur either positively or negatively but not both. ${ }^{10} \mathrm{~A} k$-clause is a clause of size at most $k$. A $k$-pclause is a positive $k$-clause, i.e., a disjunction where all literals are positive, and a $k$-nclause is a negative $k$-clause, where all literals are negative. Given a clause $\varphi=\ell_{1} \vee \cdots \vee \ell_{k}$, we say that an issue $j$ occurs in $\varphi$ if one and only one of $p_{j}$ and $\neg p_{j}$ is one of the literals of $\varphi$. For instance, the following formula $p \vee q \vee \neg p \vee \neg r \vee \neg r$ is a 2-clause in which two propositional symbols $q$ and $r$ occur, while $p$ occurs in a spurious way and does not add to the length of the clause.

\subsection{Positive and Negative Clauses}

We start by studying the special case of positive and negative clauses of arbitrary size, obtaining necessary and sufficient conditions for quota rules to lift such constraints, and exploring characterisation results inside these classes.

Proposition 21. A quota rule is $C R$ for an exact $k$-pclause IC if and only if $\sum_{j} q_{j}<n+k$, with $j$ ranging over all issues that occur in IC and $n$ being the number of individuals, or $q_{j}=0$ for at least one

\footnotetext{
${ }^{10}$ This is to exclude from the count redundant subformulas of a clause like $p_{j} \vee p_{j}$ or $p_{j} \vee \neg p_{j}$.
} 
issue $j$ that occurs in IC.

Proof. Suppose IC $=p_{1} \vee \cdots \vee p_{k}$ and call $i_{1}, \ldots, i_{k}$ the corresponding issues. Given that IC is a positive clause, the only way to generate a paradox is by rejecting all issues $i_{1}, \ldots, i_{k}$. It is easy to see that this cannot occur if the quota for one of these issues is 0 . We can therefore assume that all quotas are positive.

Suppose now that we can create a paradoxical profile $\boldsymbol{B}$. Every individual ballot $B_{i}$ must accept at least one issue to satisfy the integrity constraint; therefore the profile $\boldsymbol{B}$ contains at least $n$ acceptances concerning issues $i_{1}, \ldots, i_{k}$. On the other hand, since $F(\boldsymbol{B})_{j}=0$ for all $j=1, \ldots k$, we have that the number of individuals accepting an issue $j$ is strictly lower than $q_{j}$. As previously remarked, there are at least $n$ acceptances on the profile $\boldsymbol{B}$ and the maximal number of acceptances that allows rejection on all issues is $\sum_{j}\left(q_{j}-1\right)$. Hence $n \leqslant \sum_{j}\left(q_{j}-1\right)$. This is equivalent to $n+k \leqslant \sum_{j} q_{j}$, since all $j$ are distinct, thus we can construct a paradox with our IC if and only if this inequality holds. By taking the contrapositive we obtain the statement of Proposition 21.

With a similar proof we get an analogous result for negative clauses. Both Propositions 21 and Proposition 22 can be obtained as corollaries of a general result we shall prove in Section 6.3 (see Theorem 30). For a more detailed proof of the following proposition we refer to our previous work (Grandi, 2012a).

Proposition 22. A quota rule is CR for an exact $k$-nclause IC if and only if $\sum_{j} q_{j}>(k-1) n$, with $j$ ranging over all issues that occur in IC and $n$ being the number of individuals, or $q_{j}=n+1$ for at least one issue $j$ that occurs in IC.

In case $k=1$, i.e., the case of aggregators lifting both literals $p_{j}$ and $\neg p_{j}$, we obtain $q_{j}<n+1$ from Proposition 21 and $q_{j}>0$ from Proposition 22, thus forcing the rule to be unanimous on issue $j$ (quota rules satisfying $1 \leqslant q_{j} \leqslant n$ for all $j$ are unanimous). This is consistent with our Theorem 10 .

We now want to turn these results into characterisation results in the line of those proved in Section 4. We first have to define languages of clauses from our definition of clauses of a limited size. Let $k$-pclauses and $k$-nclauses denote, respectively, the language for integrity constraints generated from positive (negative) clauses of size $\leqslant k$. Denote by $\mathcal{Q R}_{e\left(q_{j}\right)}$ the set of quota rules such that all quotas $q_{j}$ for $j \in \mathcal{I}$ satisfy the equation in the subscript. The function $\lceil x\rceil$ is defined as the smallest integer greater than or equal than $x$. We prove the following corollary of Propositions 21 and 22:

Corollary 23. The following inclusions are true:

(i) $\mathcal{Q R}_{q_{j} \leqslant\left\lceil\frac{n}{k}\right\rceil} \subseteq \mathcal{C} \mathcal{R}[k$-pclauses $]$

(ii) $\mathcal{Q R}_{q_{j} \geqslant n-\left\lceil\frac{n}{k}\right\rceil+1} \subseteq \mathcal{C R}[k$-nclauses $]$

Proof. The first result is a consequence of Proposition 21 and the fact that $\left\lceil\frac{n}{k}\right\rceil<\frac{n}{k}+1$. If all quotas $q_{j} \leqslant\left\lceil\frac{n}{k}\right\rceil$ then for any subset of $k$ issues that might occur in a $k$-pclause we have that $\sum_{j} q_{j} \leqslant \sum_{j}\left\lceil\frac{n}{k}\right\rceil<$ $\sum_{j}\left(\frac{n}{k}+1\right)=n+k$.

Analogously, referring this time to Proposition 22, we have that for any set of $k$ issues $\sum_{j} q_{j} \geqslant$ $\sum_{j}\left(n-\left\lceil\frac{n}{k}\right\rceil+1\right)>\sum_{j}\left(n-\frac{n}{k}-1+1\right)=(k-1) n$.

The significance of the previous result resides in the fact that the bounds given by those equations are the lowest uniform bounds we can give to quotas to guarantee collective rationality, as we prove in the following corollary:

Corollary 24. A uniform quota rule is $C R$ with respect to:

(i) a k-pclause if and only if $q \leqslant\left\lceil\frac{n}{k}\right\rceil$;

(ii) a k-nclause if and only if $q \geqslant n-\left\lceil\frac{n}{k}\right\rceil+1$.

Proof. (i) In the case of uniform quota rules the equation in Proposition 21 takes the following form: $\sum_{j} q=k q<n+k$. This holds if and only if $q<\frac{n}{k}+1$, which is equivalent, as remarked in the proof of the previous corollary, to $q \leqslant\left\lceil\frac{n}{k}\right\rceil$.

(ii) In the same way, using a single quota in the equation of Proposition 22 we obtain $k q>(k-1) n$, i.e., $q>n-\frac{n}{k}$ which is equivalent to $q \geqslant n-\left\lceil\frac{n}{k}\right\rceil+1$. 
Note that the two equations in the previous proposition are incompatible, except for the cases of $k=1$, as observed after Proposition 22, and $k=2$ with $n$ being odd, in which case $q=\frac{n+1}{2}$. This proves that no uniform quota rule is collectively rational on both positive and negative clauses of a given size larger than 2, except for the case of $n$ odd and $q=\frac{n+1}{2}$. This quota rule is known as the majority rule, and it is now time to study this procedure in more detail.

\subsection{Clauses of Size 2: The Majority Rule}

The majority rule is the uniform quota rule that accepts an issue whenever there are more individuals accepting the issue than rejecting it. The majority rule is perhaps one of the most natural aggregation rules. It is arguably the one that is used most in practical applications, but as we noted in Section 1, it also generates a plethora of paradoxical situations that have been widely studied in the literature.

In case the number of individuals is odd, the majority rule has a unique definition by setting the quota to $q=\frac{n+1}{2}$. As we have seen in Section 2.5, in this case the majority rule is axiomatised by A, I, $\mathrm{M}^{\mathrm{I}} \mathrm{N}^{\mathcal{I}}$ and $\mathrm{N}^{\mathcal{D}}$ for the case of more than two issues. The case of an even number of individuals is more problematic, to account for profiles in which exactly half of the individuals accept an issue and exactly half reject it. We give two different definitions. The weak majority rule with quota $q=\frac{n}{2}$ accepts an issue if and only if at least half of the individuals accepts it. The strict majority rule accept an issue if and only if a strict majority of the individuals accept it, i.e., it is the uniform quota rule with quota $q=\frac{n+2}{2}$. The first rule favours acceptance, while the second favours rejection of an issue. In the following sections we will characterise for each of the two cases the set of integrity constraints that are lifted by the corresponding definition of the majority rule.

\subsubsection{Odd Number of Individuals: The Majority Rule}

In this section we make the assumption that the number of individuals is odd, and we indicate with $M a j$ the uniform quota rule with quota $q=\frac{n+1}{2}$. We make the additional assumption that there are at least 3 individuals. The majority rule in the case of 1 individual is the identity function that outputs the ballot received by the only individual.

Let us begin with a base-line result that proves collective rationality of the majority rule in case the integrity constraint is equivalent to a conjunction of 2-clauses:

Proposition 25. The majority rule is in $\mathcal{C R}$ [2-clauses].

Proof. Let us first consider the case of a single 2-clause IC $=\ell_{j} \vee \ell_{k}$, where $\ell_{j}$ and $\ell_{k}$ are two literals, i.e., atoms or negated atoms. A paradoxical profile for the majority rule with respect to this integrity constraint features a first majority of individuals not satisfying literal $\ell_{j}$, and a second majority of individuals not satisfying literal $\ell_{k}$. By the pigeonhole principle these two majorities must have a nonempty intersection, i.e., there exists one individual that does not satisfy both literals $\ell_{j}$ and $\ell_{k}$, but this is incompatible with the requirement that all individual ballots satisfy IC. To conclude the proof, it is sufficient to observe that if IC is equivalent to a conjunction of two clauses, then all individuals satisfy each of these clauses, and by the previous discussion all these clauses will also be satisfied by the outcome of the majority rule.

An easy corollary of this proposition covers the case of just 2 issues:

Corollary 26. If $|\mathcal{I}| \leqslant 2$, then the majority rule is in $\mathcal{C} \mathcal{R}\left[\mathcal{L}_{P S}\right]$.

Proof. This follows immediately from Proposition 25 and Lemma 3. Every given IC for two issues can be put in conjunctive normal form. Since the domain features at most two issues there are only two propositional symbols in the propositional language and thus the normal form consists of a conjunction of clauses of size at most 2 . 
Many of the classical paradoxes involving the majority rule can be formalised in our framework by means of an integrity constraint that consists of (or is equivalent to) one or more clauses with size bigger than two (Grandi, 2012b). As an example, consider the requirement of transitivity in preference aggregation, that in our framework is the integrity constraint $p_{a b} \wedge p_{b c} \rightarrow p_{a c}$, which is equivalent to a clause of size 3 . The paradoxical situation we described in the introduction constitutes another example, in which by using an exact 3-clause $\neg T_{1} \vee \neg T_{2} \vee A$ as a rationality assumption we derived a paradoxical situation using the majority rule. We now generalise this observation to a theorem that completes the characterisation of the integrity constraints lifted by the majority rule, proving that these are all and only those formulas that are expressible as conjunctions of 2-clauses. We need some preliminary definitions and a lemma.

Call a minimally falsifying partial assignment (mifap-assignment) for an integrity constraint IC an assignment to some of the propositional variables that cannot be extended to a satisfying assignment, although each of its proper subsets can.

We first prove a a crucial lemma about mifap-assignments. Given a propositional formula $\varphi$, associate with each mifap-assignment $\rho$ for $\varphi$ a conjunction $C_{\rho}=\ell_{1} \wedge \cdots \wedge \ell_{k}$, where $\ell_{i}=p_{i}$ if $\rho\left(p_{i}\right)=1$ and $\ell_{i}=\neg p_{i}$ if $\rho\left(p_{i}\right)=0$ for all propositional symbols $p_{i}$ on which $\rho$ is defined. The conjunction $C_{\rho}$ represents the mifap-assignment $\rho$ and it is clearly inconsistent with $\varphi .^{11}$

Lemma 27. Every non-tautological formula $\varphi$ is equivalent to $\left(\bigwedge_{\rho} \neg C_{\rho}\right)$ with $\rho$ ranging over all mifapassignments of $\varphi$.

Proof. Let $A$ be a total assignment for $\varphi$. Suppose $A \not \models \varphi$, i.e., $A$ is a falsifying assignment for $\varphi$. Since $\varphi$ is not a tautology there exists at least one such $A$. By sequentially deleting propositional symbols from the domain of $A$ we eventually find a mifap-assignment $\rho_{A}$ for $\varphi$ included in $A$. Hence, $A$ falsifies the conjunct associated with $\rho_{A}$, and thus the whole formula $\left(\bigwedge_{\rho} \neg C_{\rho}\right)$.

Assume now $A \models \varphi$ but $A \not \models\left(\bigwedge_{\rho} \neg C_{\rho}\right)$. Then there exists a $\rho$ such that $A \models C_{\rho}$. This implies that $\rho \subseteq A$, as $C_{\rho}$ is a conjunction. Since $\rho$ is a mifap-assignment for $\varphi$, i.e., it is a falsifying assignment for $\varphi$, this contradicts the assumption that $A \models \varphi$.

We are now able to provide a full characterisation of the set of integrity constraints that are lifted by the majority rule in case the set of individuals is odd: ${ }^{12}$

Theorem 28. $\mathcal{L} \mathcal{F}[M a j]=2$-clauses

Proof. One direction is entailed by Proposition 25: the majority rule is CR with respect to conjunctions of 2-clauses.

For the opposite direction assume that IC $\notin$ 2-clauses, i.e., that IC is not equivalent to a conjunction of 2-clauses. We now build a paradoxical profile for the majority rule. By Lemma 27 we know that IC is equivalent to the conjunction $\bigwedge_{\rho} \neg C_{\rho}$ of all mifap-assignments $\rho$ for IC. We can therefore infer that at least one mifap-assignment $\rho^{*}$ has size $>2$, for otherwise IC would be equivalent to a conjunction of 2-clauses.

Consider now the following profile. Let $y_{1}, y_{2}, y_{3}$ be three propositional variables that are fixed by $\rho^{*}$. Assume that there are at least 3 individuals. Let the first individual $i_{1}$ accept the issue associated with $y_{1}$ if $\rho\left(y_{1}\right)=0$, and reject it otherwise, i.e., let $b_{1,1}=1-\rho^{*}\left(y_{1}\right)$. Furthermore, let $i_{1}$ agree with $\rho^{*}$ on the remaining propositional variables. By minimality of $\rho^{*}$, this partial assignment can be extended to a satisfying assignment for IC, and let $B_{i_{1}}$ be such an assignment. Repeat the same construction for individual $i_{2}$, this time changing the value of $\rho^{*}$ on $y_{2}$ and extending it to a satisfying assignment to

\footnotetext{
${ }^{11}$ The notion of mifap-assignment corresponds to what are called minimally inconsistent sets in the judgment aggregation literature (List and Puppe, 2009). For a detailed discussion of the relationship between binary aggregation and judgment aggregation, we refer to our previous work (Grandi and Endriss, 2011; Grandi, 2012a). Formulas $\neg C_{\rho}$ associated with mifap-assignments $\rho$ for IC are also known as the prime implicates of IC (Marquis, 2000). Lemma 27 is a reformulation of the known result that a formula is equivalent to the conjunction of its prime implicates.

${ }^{12}$ This result may be considered a "syntactic counterpart" of a result by Nehring and Puppe (2007), in which it is proved that in the framework of judgment aggregation the majority rule will output a consistent outcome if and only if the set of formulas under consideration satisfies what is called the median property, i.e., that no minimally inconsistent subsets of size $\geqslant 3$ can be constructed from such formulas.
} 
obtain $B_{i_{2}}$. The same construction for $i_{3}$, changing the value of $\rho^{*}$ on issue $y_{3}$ and extending it to a satisfying assignment $B_{i_{3}}$. If there are other individuals in $\mathcal{N}$, let individuals $i_{3 s+1}$ have the same ballot $B_{i_{1}}$, individuals $i_{3 s+2}$ ballot $B_{i_{2}}$ and individuals $i_{3 s+3}$ ballot $B_{i_{3}}$. The basic profile for 3 issues and 3 individuals is shown in Table 6.2.1.

\begin{tabular}{llll} 
& $y_{1}$ & $y_{2}$ & $y_{3}$ \\
\hline$i_{1}$ & $1-\rho^{*}\left(y_{1}\right)$ & $\rho^{*}\left(y_{2}\right)$ & $\rho^{*}\left(y_{3}\right)$ \\
$i_{2}$ & $\rho^{*}\left(y_{1}\right)$ & $1-\rho^{*}\left(y_{2}\right)$ & $\rho^{*}\left(y_{3}\right)$ \\
$i_{3}$ & $\rho^{*}\left(y_{1}\right)$ & $\rho^{*}\left(y_{2}\right)$ & $1-\rho^{*}\left(y_{3}\right)$ \\
\hline Maj & $\rho^{*}\left(y_{1}\right)$ & $\rho^{*}\left(y_{2}\right)$ & $\rho^{*}\left(y_{3}\right)$
\end{tabular}

Table 2: A paradoxical profile for the majority rule.

As can be seen from the table, and easily generalised to the case of more than 3 individuals, there is a majority supporting $\rho^{*}$ on every variable on which $\rho^{*}$ is defined. Since $\rho^{*}$ is a mifap-assignment and therefore cannot be extended to an assignment satisfying IC, the majority rule in this profile is not collectively rational with respect to IC.

Recall that this result does not give rise to a characterisation result, i.e., it does not imply that $\mathcal{C}$ R $[2$-clauses] $=$ $\{M a j\}$ (cf. Proposition 9). On the contrary, the class $\mathcal{C} \mathcal{R}[2$-clauses] includes all generalised dictatorships. We will provide a characterisation of this class inside the class of quota rules in Section 6.3.

\subsubsection{Even Number of Individuals: Weak Majority and Strict Majority}

If the aggregation problem features an even number of individuals the majority rule can take two forms, as we have observed at the beginning of Section 6.2. Recall that the weak majority rule ( $W-M a j)$ is the uniform quota rule with quota $q=\frac{n}{2}$ while the strict majority rule $(S-M a j)$ has quota $q=\frac{n+2}{2}$.

The main difference to an odd number of individuals is that both the weak and the strict majority rule do not satisfy the axiom of domain-neutrality $\mathrm{N}^{\mathcal{D}} .{ }^{13}$ For what concerns the behaviour with respect to collective rationality, we can prove the following proposition:

Proposition 29. $W$-Maj and S-Maj are CR with respect to $\mathcal{L}_{\rightarrow}^{+}$(i.e., 2-clauses in which one literal is negative and one is positive). W-Maj is CR with respect to 2-pclauses. S-Maj is CR with respect to 2-nclauses.

Proof. A closer inspection of the proof of Proposition 25 reveals that the case for mixed clauses is also applicable for an even number of individuals. Equivalently, we could get the same result from Theorem 14, since both $W-M a j$ and $S$-Maj satisfy issue-neutrality and neutrality-monotonicity. The second part of the proposition is a direct consequence of Corollary 24 .

Unfortunately, a result analogous to Theorem 28 for the case of an even number of individuals cannot be proved. We therefore refer to the more general result about uniform quota rules proved in the following section (Corollary 31).

\subsection{General Clauses}

In this section we present a general result for the collective rationality of an arbitrary quota rule with respect to an arbitrary $k$-clause. This result generalises our previous results concerning positive and negative clauses, and clauses of size 2 . At the end of the section we prove some conclusive characterisations for collectively rational procedures inside the class of quota rules. We prove the following general result for arbitrary $k$-clauses: ${ }^{14}$

\footnotetext{
${ }^{13}$ Since they are both uniform quota rules, they still satisfy $\mathrm{I}, \mathrm{A}, \mathrm{N}^{\mathcal{I}}$ and $\mathrm{M}^{\mathrm{I}}$.

${ }^{14}$ This proposition corresponds to a result proved by Dietrich and List (2007b, Theorem 2c) for quota rules in the framework of judgment aggregation.
} 
Theorem 30. A quota rule is $C R$ with respect to an exact $k$-clause IC if and only if

$$
\sum_{j \text { negative }} q_{j}+\sum_{j \text { positive }}\left(n-q_{j}+1\right)>n(k-1)
$$

for issues $j$ that occur positively or negatively in $\mathrm{IC}$, or $q_{j}=0$ for some issue $j$ that occurs positively in IC, or $q_{j}=n+1$ for issue $j$ that occurs negatively in IC.

Proof. The case of quota rules being constant on one of the issues (i.e., the case $q_{j}=0, n+1$ ) is straightforward. We can therefore assume that all quotas are $0<q_{j}<n+1$. Suppose now that we can generate a paradoxical profile $\boldsymbol{B}$ for the $k$-clause IC. The only way to falsify the integrity constraint is to output an assignment $F(\boldsymbol{B})$ that rejects all issues that occur positively in IC and accepts all the ones that are negative. We therefore concentrate our attention to the subprofile $\boldsymbol{B}^{k}$ defined by restricting the individual ballots to the $k$ issues occurring in IC.

Since individual ballots are rational, there are at least $n$ "correct" symbols in this subprofile, i.e., a 1 for a positive issue or a 0 for a negative one. We refer to such entries with a $\mathrm{C}$ in Table 6.3. We now want to count how many "wrong" symbols are present in this subprofile. As $\boldsymbol{B}$ is a paradoxical profile, all issues that occur negatively in IC have to be accepted. Therefore, for each of those issues at least $q_{j}$ individuals have the wrong symbol, in this case a 1 . For the same reason, every issue that occurs positively in IC is rejected, so the profile $\boldsymbol{B}^{k}$ contains at least $n-q_{j}+1$ individuals rejecting such issue.

\begin{tabular}{lllll} 
& $j_{1}$ & $j_{2}$ & $\ldots$ & $j_{k}$ \\
\hline$i_{1}$ & $\mathrm{C}$ & $\mathrm{W}$ & $\ldots$ & $\mathrm{W}$ \\
$i_{2}$ & $\mathrm{~W}$ & $\mathrm{~W}$ & $\ldots$ & $\mathrm{C}$ \\
$\vdots$ & $\vdots$ & $\vdots$ & & $\vdots$ \\
$i_{n}$ & $\mathrm{~W}$ & $\mathrm{C}$ & $\ldots$ & $\mathrm{W}$ \\
\hline$F$ & $\mathrm{~W}$ & $\mathrm{~W}$ & $\ldots$ & $\mathrm{W}$
\end{tabular}

Table 3: A paradoxical profile for general clauses.

Summing up, since the number of cells in this subprofile is $n k$, we can generate a paradoxical profile if and only if there are enough cells in $\boldsymbol{B}^{k}$ to account for the minimal number of correct and wrong symbols to generate a paradox. This turns into the equation $n+\sum_{j \text { pos }}\left(n-q_{j}+1\right)+\sum_{j n e g} q_{j} \leqslant n k$. By taking the contrapositive of the last statement we get Equation 1 .

It is easy to see that the special cases of positive and negative clauses, i.e., our Propositions 21 and 22, can be obtained as corollaries of Theorem 30. The special case of uniform quota rules is particularly interesting:

Corollary 31. A uniform quota rule with $q \neq 0, n+1$ is $C R$ with respect to a $k$-clause IC if and only if

$$
\left(k_{2}-k_{1}\right) q>n\left(k_{2}-1\right)-k_{1}
$$

where $k_{1}$ (respectively $k_{2}$ ) is the number of positive (negative) issues in IC.

Our Corollary 24 can be proved as the special case of $k_{1}=k$ and $k_{1}=0$, respectively. The other special case of $k_{1}=k_{2}$ leads to a satisfiable equation only in case of $k=2$, proving two important facts: First, every uniform quota rule lifts a 2-clause in which one issue is positive and the other is negative, for the equation in this case is always true (it reduces to $0>-1$ ). More importantly, it implies that $\mathcal{C R}[k$-clauses] does not contain any uniform quota rule for $k>3$ when the number of issues is even, since this language includes also $k$-clauses where exactly half of the issues are negative and half are positive, in which case the equation does not have any solutions.

We are now ready to prove a characterisation analogous to that of Theorem 28 for uniform (nonconstant) quota rules: 
Proposition 32. Let $q$ be different from $0, n+1$, and let $F_{q}$ be the corresponding uniform quota rule. Then, $\mathcal{L} \mathcal{F}\left[F_{q}\right]$ is the language for integrity constraints generated from all $k$-clauses that satisfy Equation 2.

Proof. Let IC be an integrity constraint. By Lemma 27, IC is equivalent to the conjunction of the negation of its mifap-assignments $\bigwedge_{\rho} \neg C_{\rho}$. Hence, $F_{q}$ is CR with respect to IC if and only if $F_{q}$ lifts each clause $C_{\rho}$, and by Corollary 31 this holds if and only if each $C_{\rho}$ satisfies Equation 2 . Thus, $F_{q}$ is CR with respect to IC if and only if IC is equivalent to a conjunction of clauses satisfying Equation 2.

Using the equations introduced in this section we are able to prove some interesting results about the characterisation of collectively rational procedures inside the class of quota rules. We have already seen some partial inclusion in Section 6.1 for the language of positive and negative clauses, and we can now prove the following:

Proposition 33. If the number of individuals is odd, then $\mathcal{C} \mathcal{R}[2$-clauses $] \cap \mathcal{Q R}=\{$ Maj $\}$

Proof. Let $q_{1}$ and $q_{2}$ be two quotas for two distinct issues. Since we assume that every 2-clause is lifted, these two quotas satisfy the following system of equations, obtained by instantiating Equation 1 to the case of positive, negative, and mixed 2-clauses:

$$
\begin{aligned}
q_{1}+q_{2}<n+2 \\
q_{1}+q_{2}>n \\
q_{1}+n-q_{2}+1>n \\
q_{2}+n-q_{1}+1>n
\end{aligned}
$$

From the first two equations we obtain $q_{1}+q_{2}=n+1$, since quotas are integers. From the other equations we obtain $\left|q_{1}-q_{2}\right|<1$, which for integer values entails $q_{1}=q_{2}$. We can then conclude that $q_{1}=q_{2}=\left\lceil\frac{n+1}{2}\right\rceil$, thus obtaining the majority rule.

We end this section by proving an expected negative result for the characterisation of general languages of clauses inside the class of quota rules:

Proposition 34. $\mathcal{C} \mathcal{R}[k$-clauses $] \cap \mathcal{Q R}=\emptyset$ for all $k>2$.

Proof. A quota rule in $\mathcal{C} \mathcal{R}[k$-clauses $]$ must be $\mathrm{CR}$ with respect to both positive and negative clauses, therefore both equations in Propositions 21 and 22 have to be satisfied. But these are unsolvable for $k>2$. To see this, consider the first equation, which forces $\sum q_{j}<n+k$ for any subsets of issues of size $k$, and the second equation requiring $\sum q_{j}>(k-1) n$ on the same subsets. These two equations are compatible only if $(k-1) n<n+k$, from which we obtain $n<\frac{k}{k-2}$. This in turn is true only if $n<3$, in contradiction to our assumption that there are at least 3 individuals.

Observe that the equations involved in this proof do not assume that clauses have size strictly smaller than $k$, hence this result can be strengthened to the language of clauses of size exactly $k$.

\section{Discussion}

In this section we shall discuss the impact of the results of the present paper on both SCT and AI, summarising also some of the results presented in our previous work (Grandi and Endriss, 2011; Grandi, 2012b,a).

Preference aggregation (Arrow, 1963) and judgment aggregation (List and Puppe, 2009) are amongst the main frameworks for aggregation that are studied in SCT and AI, and the relation between these two frameworks is the object of study of several publications (Dietrich and List, 2007a; Grossi, 2009; Porello, 2010). As previously shown in Examples 1 and 2, both frameworks can be interpreted as instances of binary aggregation by devising suitable integrity constraints. This fact already constitutes a contribution 
in itself, showing that our setting is a truly general framework for the study of collective rationality in aggregation theory.

Classical studies in these frameworks have focused on the observation of paradoxical situations and on proving so-called impossibility results, aimed at showing the impossibility of non-paradoxical aggregation under seemingly natural axiomatic conditions. It can be easily seen that classical paradoxes, such as the Condorcet paradox in preference aggregation and the discursive dilemma in judgment aggregation (Kornhauser and Sager, 1986), can be viewed as instances of our Definition 4, which stands out as a general definition of paradox in aggregation theory (Grandi, 2012b). Moreover, as we shall explain next, the generality of our framework enables us to develop a new proof method for (im)possibility results in SCT. Classical work in SCT studies the existence of aggregation procedures in a given framework for aggregation, once a set of desirable conditions have been identified in a list of axiomatic properties. By translating such aggregation problems into binary aggregation, we are able to identify the source of impossibilities in a clash between the integrity constraint that defines the framework and the axiomatic properties that are being required, making use of characterisation results such as those we presented in this paper. The results that can be obtained by using this proof method may share similarities or may be weaker than known results from the literature on SCT, especially for the case of independent aggregation rules. However, the focus is not on the novelty or strength of specific results, but rather on the generality and flexibility of the proof method we put forward. By unifying proofs in aggregation theory we gain a deeper understanding of the common problem behind many classical results: impossibilities arise from clashes between axiomatic properties and requirements of collective rationality.

We provided several examples of the use of this method in previous work (Grandi and Endriss, 2011; Grandi, 2012a), and we now sketch one such finding for the case of preference aggregation. Recall that aggregation procedures in preference aggregation are called social welfare functions (Arrow, 1963), and that they associate a collective preference with every profile of individual orders over a set of alternatives $\mathcal{X}$. Axiomatic properties such as those presented in Section 2.4 can be devised for social welfare functions, and we refer to the relevant literature for their precise definition (Gaertner, 2006). We call a social welfare function imposed if for some pair of distinct alternatives $a$ and $b$ we have that $a$ is always collectively preferred to $b$ in every profile. We show the following proposition (a more detailed proof can be found in our previous work (Grandi and Endriss, 2011; Grandi, 2012a):

Proposition 35. If $|\mathcal{X}| \geqslant 3$ and $|\mathcal{N}| \geqslant 2$, then any anonymous, independent and monotonic social welfare function for $\mathcal{X}$ and $\mathcal{N}$ is imposed.

Proof. The first step is to move from preference aggregation to binary aggregation, as outlined in our Example 2 of Section 2.2. This embedding can be extended to aggregation procedures: every anonymous, independent and monotonic social welfare function corresponds to a binary aggregation procedure that is collectively rational for $\mathrm{IC}_{<}$and that satisfies $\mathrm{A}$, I and $\mathrm{M}^{\mathrm{I}}$. Recall that by Proposition 1, every aggregation procedure satisfying $\mathrm{A}, \mathrm{I}$ and $\mathrm{M}^{\mathrm{I}}$ is a quota rule.

The second step consists in checking whether the axiomatic requirements clash with the integrity constraint under consideration. In this case the answer is positive: by exploiting some of the characterisation results proved in Section 6 we now prove that, if a quota rule is collectively rational for $\mathrm{IC}_{<}$, then it is imposed, i.e., at least one of the quotas $q_{a b}$ is equal to 0. Suppose, for the sake of contradiction, that every quota $q_{a b}>0$. For any three alternatives $a, b, c \in \mathcal{X}$, the integrity constraints corresponding to transitivity can be shown to be equivalent to $p_{b a} \vee p_{c b} \vee p_{a c}$ and $p_{a b} \vee p_{b c} \vee p_{c a}$. These are positive clauses of size 3 ; thus, by Proposition 21 we obtain the following inequalities on quotas: $q_{b a}+q_{c b}+q_{a c}<n+3$ and $q_{a b}+q_{b c}+q_{c a}<n+3$. Furthermore, the requirements of completeness and antisymmetry of linear orders force the quotas to satisfy the following additional equations: $q_{a b}+q_{b a}=n+1, q_{b c}+q_{c b}=n+1$ and $q_{a c}+q_{c a}=n+1$. Now, adding the two inequalities we obtain that $\sum_{a, b \in \mathcal{X}} q_{a b}<2 n+6$ and adding the three equalities we obtain $\sum_{a, b \in \mathcal{X}} q_{a b}=3 n+3$. The two constraints together admit a solution only if $n<3$. Thus, it remains to analyse the case of 2 individuals; but it is easy to see that our constraints do not admit a solution in positive integers for $n=2$. This shows that there must be a quota $q_{a b}=0$ for certain distinct $a$ and $b$ as soon as $n \geqslant 2$; hence, the quota rule is imposed. 
Finally, going back to the initial problem, we can conclude that every anonymous, independent and monotonic social welfare function for more than 3 alternatives and 2 individuals is imposed.

Similar results can be obtained in the framework of judgment aggregation. In previous work we were able to strengthen a known characterisation of safe agendas (Endriss et al., 2010) by moving to the realm of binary aggregation and by developing syntactic analogues of the conditions that guarantee consistent aggregation of judgments on a given set of propositional formulas (Grandi and Endriss, 2011; Grandi, 2012a).

One further reason supporting the use of binary aggregation with integrity constraints in applications related to $\mathrm{AI}$ has to do with considerations of computational complexity. In recent years the framework of judgment aggregation has received increasing attention in the AI community, especially in its applications to the modelling of multiagent systems. In previous work we presented a preliminary comparison of the computational complexity of both frameworks for a number of basic tasks, showing that binary aggregation is at most as hard as classical judgment aggregation, and it is significantly easier in some situations (Grandi, 2012a). The most simple example is the problem of checking the rationality of a given ballot: While for binary aggregation this problem can be solved in polynomial time using model checking, in judgment aggregation the same problem corresponds to the satisfiability of the set of propositional formulas accepted by one of the agents, a much harder problem which is known to be NP-complete.

Another interesting application is suggested by our Theorem 16. This result identifies an attractive class of aggregation procedures in the class of generalised dictatorships, obtaining procedures that lift all integrity constraints expressible in the language of propositional logic. Interesting procedures can therefore be defined by deciding which of the individual ballots best represent the ballots submitted in a given profile. Inspired by related work in the literature on belief merging (Konieczny and Pino Pérez, 2002 ), in previous work we introduced a new aggregation rule which given a profile $\boldsymbol{B}$ selects those individual ballots that minimise the sum of the Hamming distances from the other individual ballots in

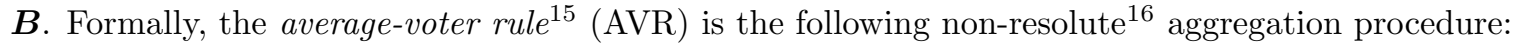

$$
\operatorname{AVR}(\boldsymbol{B})=\underset{\left\{B_{i} \mid i \in \mathcal{N}\right\}}{\operatorname{argmin}} \sum_{i^{\prime} \in \mathcal{N}} H\left(B_{i}, B_{i^{\prime}}\right),
$$

where $H\left(B, B^{\prime}\right)=\sum_{j \in \mathcal{I}}\left|b_{j}-b_{j}^{\prime}\right|$ is the Hamming distance between binary ballots. Besides being collectively rational for every rationality assumption, this rule also enjoys interesting axiomatic and computational properties (Grandi and Endriss, 2011; Grandi, 2012a). This rule adds to a growing literature on the development of aggregation procedures for binary and judgment aggregation inspired by AI techniques (Pigozzi et al., 2009; Lang et al., 2011).

\section{Related Work}

While the framework of binary aggregation is well-known in the literature on SCT, this paper is the first systematic study of collective rationality with respect to languages for integrity constraints in this setting. Moreover, classical approaches concentrate on Arrovian aggregation procedures, i.e., procedures that are both unanimous and independent, while most of our characterisation results do not make such restrictive assumptions. As we have argued before, while the restriction to Arrovian aggregators is in line with standard assumptions in economics, those assumptions are not always justified in AI applications. In this section we review some of the classical approaches to the problem of collective rationality that can be found in the literature.

Wilson (1975) has been the first to define and study the framework of binary aggregation, in order to obtain an impossibility result for independent aggregation procedures that generalises the more famous result by Arrow (1963). This setting has been investigated more recently by Dokow and Holzman (2010a)

\footnotetext{
${ }^{15}$ This rule is called distance-based generalised dictatorship in our previous work (Grandi and Endriss, 2011).

${ }^{16}$ That is, it associates with every profile a set of binary ballots rather than a single one. Non-resolute aggregators can be made resolute by the introduction of a tie-breaking rule, e.g., a lexicographic ordering over the domain $\mathcal{D}$.
} 
and Nehring and Puppe (2010). In the former work, the authors study the existence of independent, unanimous and non-dictatorial procedures that are collectively rational with respect to certain domain restrictions. As we have remarked at the beginning of this section, our work differs in that we do not concentrate on independent aggregation procedures. Moreover, we exploit our representation of constraints as propositional formulas by defining languages for integrity constraints in a syntactic way, while Dokow and Holzman (2010a) use a model-based approach: they assume that the set of rational ballots is specified explicitly.

Results in line with our work of Section 6 can be obtained by restricting the set of aggregation procedures to independent rules alone, focusing on the study of winning coalitions, i.e., those subsets of $\mathcal{N}$ that can force acceptance on a given issue. Results in this setting have been proven in the case of judgment aggregation by Nehring and Puppe (2007) and generalised by Dietrich and List (2007b), giving conditions on the structure of winning coalitions to guarantee collective rationality.

As we already remarked in several places throughout the paper, many of the results on quota rules proved in Section 6 are analogous to those proved by Dietrich and List (2007b) in the framework of judgment aggregation. The use of integrity constraints, however, enables us to use syntax to analyse possible sources of impossibility, resulting in a flexible framework that is arguably easier to implement.

A related field of research is that of belief merging (Konieczny and Pino Pérez, 2002, 2011), in which sets of propositional formulas are aggregated into a collective set. The two frameworks share interesting features, but study different (although related) problems. While binary aggregation imposes an integrity constraint as a rationality assumption for both individual and collective outcomes, in belief merging integrity constraints are enforced only on the outcome. This reflects the view that the integrity constraint is a feasibility requirement rather than a rationality assumption, and should not be enforced on individual preferences or beliefs. Nevertheless, exploring possible ways of combining the two settings constitutes an interesting direction for future work.

\section{Conclusions and Perspectives}

In this paper we have presented a general framework for the study of collective rationality in aggregation theory. Our framework is based on binary aggregation, in which a group of individuals each express a ballot in a binary multi-issue domain, and these ballots are then aggregated into a collective one. The generality of this setting allowed us to model important problems in multiagent systems such as multi-issue elections, preference aggregation, judgment aggregation, and the problem of choosing from a set of candidates. We have formalised rationality assumptions using a simple propositional language, which enables us to classify such formulas in a syntactic way. Depending on the syntactic properties of the rationality assumptions at hand we have then investigated the problem of collective rationality: how can we guarantee, by means of classical axiomatic properties, that the outcome of the aggregation satisfies the same rationality assumption as the individual ballots? We have also studied the opposite problem: we have characterised, given a classical axiomatic requirement for aggregation procedures, the set of integrity constraints that are lifted by all procedures satisfying such properties. In the last part of the paper we have concentrated on quota rules, i.e., procedures defined by means of acceptance quotas for every issue, and especially on the majority rule, obtaining complete characterisations of the set of integrity constraints lifted by such procedures. Our results provide a systematic answer to the question of recognising domains of aggregation over which paradoxes can be avoided, reducing this problem to the syntactical analysis of the propositional formulas that define rationality assumptions. Not only does this allow for a uniform analysis of such diverse problems as preference aggregation, judgment aggregation and multi-issue elections in general, but it also has the advantage of expressing properties in a computationfriendly language such as propositional logic.

This paper constitutes a first step towards a general application-oriented theory of aggregation, a topic that is crucial to the development of several AI applications and, above all, to the design of multiagent systems. The main achievements of this paper are twofold: First, this work constitutes the first systematic study of collective rationality for non-independent aggregation procedures in binary aggregation. While 
the literature in SCT has traditionally focused on independent aggregation procedures for preferences or judgments, we provided a truly general setting in which classical frameworks can be interpreted and new results can be provided. Second, to achieve these results we introduced the novel concept of languages for integrity constraints. By developing a complex theoretical machinery around this notion we were able to build a link between classical axiomatic properties and collective rationality, proving interesting characterisations and laying the basis for further investigations of aggregation theory.

We conclude by pointing out at some possible directions for future work. The framework we developed in this paper can be employed to deepen the current analysis of voting in multi-issue domains, e.g., by developing new voting methods based on preferential dependencies. In this respect, partial achievements have been made by Lang and Xia (2009); Conitzer et al. (2011); Xia et al. (2011) and, in a similar setting, in our previous work (Airiau et al., 2011). The generality of the framework we proposed in this paper may also suggest that the aggregation of logical structures represents a promising area for future work. We have recently made an initial step in this direction by providing a first study of graph aggregation (Endriss and Grandi, 2012). A similar problem is that of extending our definitions to cover the case of non-binary issues, e.g. by allowing individuals to abstain on certain issues. Related work has been done in this respect by Dokow and Holzman (2010b). The results proved in the present paper should not be interpreted as limiting the possibility of consistent aggregation, but rather as specifying for each application at hand the right conditions that make it possible. Perhaps the most intriguing direction for future research is to employ the machinery developed in this paper to design collectively rational aggregation procedures to tackle complex problems of aggregation that occur in AI applications.

\section{Acknowledgments}

We wish to thank Daniele Porello and the whole COMSOC group at the University of Amsterdam for fruitful and enthusiastic discussions. We would also like to thank the anonymous reviewers for AAAI-2010, IJCAI-2011 and the Artificial Intelligence journal, as well as the audiences of workshop and seminar talks we have given in Amsterdam, Bucharest, Dagstuhl, Delft, Estoril, Freudenstadt, Groningen, Kraków, Milan, Paris and Venice for their useful comments.

\section{References}

S. Airiau, U. Endriss, U. Grandi, D. Porello, J. Uckelman, Aggregating dependency graphs into voting agendas in multi-issue elections, in: Proceedings of the 22nd International Joint Conference on Artificial Intelligence (IJCAI-2011).

A. Altman, M. Tennenholtz, Axiomatic foundations for ranking systems, Journal of Artificial Intelligence Research 31 (2008) 473-495.

K.J. Arrow, Social Choice and Individual Values, John Wiley \& Sons, 2nd edition, 1963.

S.J. Brams, P.C. Fishburn, Approval voting, Springer, 2nd edition, 2007.

F. Cariani, M. Pauly, J. Snyder, Decision framing in judgment aggregation, Synthese 163 (2008) 1-24.

Y. Chevaleyre, U. Endriss, J. Lang, N. Maudet, A short introduction to computational social choice, in: Proceedings of the 33rd Conference on Current Trends in Theory and Practice of Computer Science (SOFSEM-2007).

Y. Chevaleyre, U. Endriss, J. Lang, N. Maudet, Preference handling in combinatorial domains: From AI to social choice, AI Magazine 29 (2008) 37-46.

V. Conitzer, J. Lang, L. Xia, Hypercubewise preference aggregation in multi-issue domains, in: Proceedings of the 22nd International Joint Conference on Artificial Intelligence (IJCAI-2011). 
F. Dietrich, C. List, Arrow's theorem in judgment aggregation, Social Choice and Welfare 29 (2007a) 19-33.

F. Dietrich, C. List, Judgment aggregation by quota rules: Majority voting generalized, Journal of Theoretical Politics 19 (2007b) 391-424.

E. Dokow, R. Holzman, Aggregation of binary evaluations, Journal of Economic Theory 145 (2010a) 495-511.

E. Dokow, R. Holzman, Aggregation of binary evaluations with abstentions, Journal of Economic Theory 145 (2010b) 544-561.

U. Endriss, U. Grandi, Graph aggregation, in: Proceedings of the 4th International Workshop on Computational Social Choice (COMSOC-2012).

U. Endriss, U. Grandi, D. Porello, Complexity of judgment aggregation: Safety of the agenda, in: Proceedings of the 9th International Joint Conference on Autonomous Agents and Multiagent Systems (AAMAS-2010).

P. Faliszewski, E. Hemaspaandra, L.A. Hemaspaandra, J. Rothe, A richer understanding of the complexity of election systems, in: S. Ravi, S. Shukla (Eds.), Fundamental Problems in Computing: Essays in Honor of Professor Daniel J. Rosenkrantz, Springer, 2009.

P. Faliszewski, A.D. Procaccia, AI's war on manipulation: Are we winning?, AI Magazine 31 (2010) $53-64$.

W. Gaertner, A Primer in Social Choice Theory, Oxford University Press, 2006.

U. Grandi, Binary Aggregation with Integrity Constraints, Ph.D. thesis, ILLC, University of Amsterdam, 2012a.

U. Grandi, The common structure of paradoxes in aggregation theory, in: Proceedings of the 4th International Workshop on Computational Social Choice (COMSOC-2012).

U. Grandi, U. Endriss, Lifting rationality assumptions in binary aggregation, in: Proceedings of the 24th AAAI Conference on Artificial Intelligence (AAAI-2010).

U. Grandi, U. Endriss, Binary aggregation with integrity constraints, in: Proceedings of the 22nd International Joint Conference on Artificial Intelligence (IJCAI-2011).

D. Grossi, Unifying preference and judgment aggregation, in: Proceedings of the 8th International Joint Conference on Autonomous Agents and Multiagent Systems (AAMAS-2009).

S. Konieczny, R. Pino Pérez, Merging information under constraints: A logical framework, Journal of Logic and Computation 12 (2002) 773-808.

S. Konieczny, R. Pino Pérez, Logic based merging, Journal of Philosophical Logic 40 (2011) 239-270.

L.A. Kornhauser, L.G. Sager, Unpacking the court, Yale Law Journal 96 (1986) 82-117.

J. Lang, Logical preference representation and combinatorial vote, Annals of Mathematics and Artificial Intelligence 42 (2004) 37-71.

J. Lang, G. Pigozzi, M. Slavkovik, L. van der Torre, Judgment aggregation rules based on minimization, in: Proceedings of the 13th Conference on Theoretical Aspects of Rationality and Knowledge (TARK2011).

J. Lang, L. Xia, Sequential composition of voting rules in multi-issue domains, Mathematical Social Sciences 57 (2009) 304-324. 
C. List, C. Puppe, Judgment aggregation: A survey, in: Handbook of Rational and Social Choice, Oxford University Press, 2009.

P. Marquis, Consequence finding algorithms, in: D. Gabbay, P. Smets (Eds.), Handbook on Defeasible Reasoning and Uncertainty Management Systems, Kluwer Academic Publishers, Dordrecht, 2000.

K.O. May, A set of independent necessary and sufficient conditions for simple majority decision, Econometrica 20 (1952) 680-684.

K. Nehring, C. Puppe, The structure of strategy-proof social choice. Part I: General characterization and possibility results on median spaces, Journal of Economic Theory 135 (2007) 269-305.

K.D. Nehring, C. Puppe, Abstract Arrowian aggregation, Journal of Economic Theory 145 (2010) 467494.

D.M. Pennock, E. Horvitz, C.L. Giles, Social choice theory and recommender systems: Analysis of the axiomatic foundations of collaborative filtering, in: Proceedings of the 17th National Conference on Artificial Intelligence (AAAI-2000).

G. Pigozzi, M. Slavkovik, L. van der Torre, A complete conclusion-based procedure for judgment aggregation, in: Proceedings of the First International Conference on Algorithmic Decision Theory (ADT2009).

M.S. Pini, F. Rossi, K.B. Venable, T. Walsh, Aggregating partially ordered preferences, Journal of Logic and Computation 19 (2009) 475-502.

D. Porello, Ranking judgments in Arrow's setting, Synthese 173 (2010) 199-210.

F. Rossi, K. Venable, T. Walsh, $m$ CP Nets: Representing and reasoning with preferences of multiple agents, in: Proceedings of the 19th AAAI Conference on Artificial Intelligence (AAAI-2004).

Y. Shoham, K. Leyton-Brown, Multiagent Systems: Algorithmic, Game-Theoretic, and Logical Foundations, Cambridge University Press, 2009.

R.B. Wilson, On the theory of aggregation, Journal of Economic Theory 10 (1975) 89-99.

L. Xia, V. Conitzer, J. Lang, Strategic sequential voting in multi-issue domains and multiple-election paradoxes, in: Proceedings of the 12th ACM Conference on Electronic Commerce (EC-2011). 